\title{
Investigation of the effect of the bed slope on extreme waves using First Order Reliability Method
}

\section{Ghadirian, Amin; Bredmose, Henrik}

Published in:

Marine Structures

Link to article, DOI:

10.1016/j.marstruc.2019.05.005

Publication date:

2019

Document Version

Early version, also known as pre-print

Link back to DTU Orbit

Citation (APA):

Ghadirian, A., \& Bredmose, H. (2019). Investigation of the effect of the bed slope on extreme waves using First Order Reliability Method. Marine Structures, 67, [102627]. https://doi.org/10.1016/j.marstruc.2019.05.005

\section{General rights}

Copyright and moral rights for the publications made accessible in the public portal are retained by the authors and/or other copyright owners and it is a condition of accessing publications that users recognise and abide by the legal requirements associated with these rights.

- Users may download and print one copy of any publication from the public portal for the purpose of private study or research.

- You may not further distribute the material or use it for any profit-making activity or commercial gain

- You may freely distribute the URL identifying the publication in the public portal

If you believe that this document breaches copyright please contact us providing details, and we will remove access to the work immediately and investigate your claim 


\title{
Investigation of the effect of the bed slope on extreme waves using First Order Reliability Method
}

\author{
Amin Ghadirian, Henrik Bredmose
}

DTU Wind Energy, Nils Koppels Alle, Bygning 403, 2800 Kgs. Lyngby, Denmark

\begin{abstract}
The effect of bed slope on the force statistics and shape of the force time history around the force peak is investigated numerically with a fully nonlinear wave model and FORM analysis. The numerical model setup is validated by comparison of averaged experimental force and free surface elevation time series and the FORM results for the given force levels. The fully nonlinear FORM solution shows a good improvement over first-order and second-order results by increasing the asymmetry of the force history through the peak. The average deviation between FORM and the experimental curve is found to be at the level of $10 \%$ of the maximum force value. Next, the order statistics for force peaks from experimental and numerical $3 \mathrm{~h}$ realization are compared. Bootstrapping is applied to estimate the expected mean value of the inline forces for a given exceedance probability and a good match between the numerical and experimental order statistics is found.

FORM predictions of the force peak histories for a cylinder on flat or sloping bed are next compared. The diameter, depth at the structure and significant wave height are kept identical between the two cases. It is found that the force histories are not changed significantly by the presence of the slope in the sea states with lower Ursell number. For the larger Ursell number sea states, the corresponding time histories of the free surface elevation, however, show a larger skewness for the flat bed case. Further, from the FORM analysis, the exceedance probabilities for a given force level at sloped bed is found to be larger than for flat bed. The ratio of exceedance probability is found to increase with force level except for the sea states with largest Ursell number where the numerical results are affected by strong nonlinearity.

The higher-harmonic content of the force histories is further analyzed by the four-phase separation method. The analysis confirms that the relative contribution from the higher-harmonic force components increases with the force peak level and further shows that the contribution from the first, second and third harmonics are very similar between flat bed and sloped bed. The analysis further highlights the presence of spurious second-harmonic waves from the linear wave generation method and shows that these are stronger for the flat bed case. The phase shift between the different harmonics of free surface elevation and inline force time series is observed to be constant for different sea states and target forces irrespective of the bed slope.

Besides the direct findings of the study, the paper demonstrates the applicability of the FORM method for determination of design waves based on certain load effects. Although difficulties may occur for breaking waves or very strong nonlinearity, the combination of FORM and a fully nonlinear wave model enables average probability levels and time histories for extreme events to be determined and applied as design waves specific to a given load level or response level.
\end{abstract}

Keywords: First Order Reliability Method, bed slope, extreme waves

\section{Introduction}

The calculation of the Ultimate Limit State (ULS) load cases is an important step in the design process of substructures for offshore wind turbines. For such cases, normally, a set of sea state characteristics are chosen from the long term data or hind cast simulations based on a target return period. Irregular time series of free surface elevation are then made using linear wave theory. Afterwards the extreme waves are selected and replaced by either linear New Wave [1] or the stream function wave theory [2] to calculate the kinematics and the distributed force on the 
structures [3]. In both methods the bed is assumed to be flat which is usually not the case in the open sea, especially close to the coast. The current study aims to investigate the effect of the presence of a sloped bed on the exceedance probability, frequency content and shape of waves that create extreme force episodes. In addition, it offers a new design wave model.

Although several studies on the effect of bed slope exist in the literature, only one study of the effect on the load on the structure exists to the authors knowledge. Nelson [4] investigated characteristics of regular waves on various slopes. These characteristics included wave profile, celerity and ratio of crest height to wave height among many others. He found out that the effect is minimal on wave celerity and potential energy but significant on crest and trough skewness and the asymmetry of the wave. Nevertheless, linked to the limitation of regular waves, he did not investigate the power spectral density or the statistical distribution of the free surface elevation of the waves on different bed slopes. Elgar and Guza [5], Freilich and Guza [6] and Freilich et al. [7] used field measurements to investigate the effect of shoaling of gravity waves and compared these results to linear shoaling theory and a nonlinear model. The nonlinear model was based on Boussinesq-type equations for a sloping bottom. The change in power spectral density of the free surface elevation of different sea states due to the slope was the focus of these studies. Hence, the time series and statistical distribution of the free surface elevation were not investigated. Nwogu [8] developed a new form of Boussinesq-type equations with the velocity variable in an arbitrary depth. These equations were then implemented and used to investigate the propagation of regular and irregular waves on a constant slope beach. Time series of the surface elevation and the power spectral density of them were investigated and the results were compared to experiments. The results demonstrated that the developed form of the equations could reasonably simulate several nonlinear effects that occur in the shoaling of surface waves from deep to shallow water. He reported that the forced lower- and higher-frequency wave harmonics amplify during shoaling and the horizontal and vertical asymmetry of the waves increases. In none of these studies the statistical distribution of the wave height and the maximum inline force were investigated.

Schløer et al. [9] investigated the statistical distribution of the maximum inline forces numerically on two bed slopes of 1:25 and 1:100. They used the same linear wave time series based on the JONSWAP spectrum at the wave generation zone, at equal depth in the two domains. The study concluded that a larger bed slope results in largest maximum crest height, inline force and bending moment. The work included numerical simulation of just one sea state. Katsardi et al. [10] used a systematic campaign of experiments to investigate the role of local water depth, peak period, significant wave height, spectral band width and the bed slope on the statistical distribution of the wave heights and the crest heights. Two different bed slopes of 1:100 and 1:250 were used. The dependence of the statistical distribution to the local effective water depth $\left(k_{p} d\right)$ and the local sea state steepness $\left(\frac{1}{2} H_{s} k_{p}\right)$ was shown and it was found that the two different mild bed slopes analyzed did not change the wave height or crest height statistical distributions. However, in this study the statistical distribution of the inline force was not investigated.

The main difference between the current work and previous literature is the focus on the extreme waves in term of maximum inline force in the present work. In this paper we present a detailed study on the effect of the bed slope on the inline force peak statistical distribution, power spectral density and the time series of the free surface elevation and inline force on two bed slopes and six sea states. Another important feature of the present study is that the sea state parameters are kept identical at the location of the structure, where results at a slope and flat bed are compared.

This study also shows the applicability of the FORM method for determination of design waves based on certain load effects. The combination of FORM and a fully nonlinear wave model enables average probability levels and time histories for extreme events to be determined and applied as design waves specific to a given load level or response level. Other design waves can also be found in the literature with different complexity of the used wave model. The most common method in the design process of offshore structures is using either the stream function wave theory [2] or the New Wave [1] combined with a stretching method. However, both of these wave theories are associated with limitations such as symmetry of the wave around the crest and flat bed assumption. In addition, the New Wave is based upon linear wave theory and cannot capture the wave non-linearities. Most recently Alberello et al. [11] performed laboratory experiments to investigate the effect of the underlying focusing mechanism on the shape of the breaking wave and its velocity field. In this regard, it was found that the shape of the wave spectrum plays a substantial role. Broader underlying wave spectra leads to energetic plungers at a relatively low amplitude. For narrower spectra waves break at higher amplitudes but with a less energetic spilling breaker. The Most Likely Wave (MLW) theory introduced by Friis-Hansen, P. \& Nielsen [12] is an extension to the New Wave theory taking into account the corresponding instantaneous long frequencies of large ocean waves. Later, Jensen [13] presented a general formulation for the 
conditional mean and its variance, which were derived for slightly non-Gaussian processes extending the model to second-order waves. The Critical Wave Episode (CWE) was suggested by Torhaug [14] is based on identification of wave candidates from the linear theory that are likely to produce extreme responses.

The CWE can be recognized easily if the transfer functions from the wave to the response are all known. The Most Likely Extreme Response (MLER) model was presented by Adegeest et al. [15], which derives the underlying wave profile that derives a conditional linear response. The method was performed using linear wave theory and linear transfer functions. Later Pastoor [16] presented the Directional Most Likely Extreme Response (DMLER) model which is an extension of the MLER model. The procedure is based on the linear model for description of a conditional response, after which the underlying wave profile may be generated with information from the linear transfer function for all wave headings. Schløer et al. [17] and Ghadirian et al. [18] used the same concept to calculate the DMLER for the inline forces on a monopile and coined the resulting theory the NewForce theory. They extended the model so that it may also include the second-order contributions. It is observed in the literature that none of the existing design waves include fully nonlinear wave theory capable of including customized bed bathymetry.

The measurements of the inline force and free surface elevation used in this study will be described in Section 2. The process used to analyze the data is explained in Section 2. In Section 3 the numerical wave model, used to simulate the fully nonlinear waves, and the First Order Reliability Method problem, solved to calculate the most probable shape of the waves with certain maximum inline force, are described. The validation of the model against measurements for cases on both flat and sloped bed are presented in Section 4. After this section only numerical results are presented. The effect of the bed slope is investigated using the proposed method in Section 5. In this section the time series of the most probable waves, their inline force time series, the spectral content and their exceedance probabilities are compared on flat and sloped bed. In Section 6 the harmonic separation method is used to separate harmonics of each wave episode on flat and sloped bed and compare the harmonic content with each other. Finally more discussions and some suggestions for the future work are presented in Section 7.

\section{Experiments and the data analysis methods}

Data from two sets of experiments are used in the present study, performed as part of the DeRisk project [19] and the Wave Loads project [20].

\subsection{DeRisk data set: flat bed}

The flat bed experiments of the DeRisk project were conducted at DHI Denmark in 2015 at a scale of 1:50. A monopile was installed on two force transducers at the top and the bottom, $7.3 \mathrm{~m}$ from the wave makers. Wave gauges were installed in the basin to measure the free surface elevation approaching and passing the monopile. In the present study only unidirectional wave sea states were investigated, even though the experiments included several directionally spread sea states. The diameter of the monopile was $7 \mathrm{~m}$ and the water depth was $33 \mathrm{~m}$ in full scale. The wave makers were of piston type and waves were generated by linear wave generation theory [21]. A schematic drawing of the wave basin layout is given in figure 1.

\subsection{Wave Loads data set: sloped bed}

The Wave Loads project [20,23], included experiments of irregular waves on a 1:25 sloped bed at scale of 1:80. The diameter of the monopile was $6 \mathrm{~m}$ and the water depth at the location of the monopile varied between $40.8 \mathrm{~m}$, $30.8 \mathrm{~m}$ and $20.8 \mathrm{~m}$ in full scale. The monopile was mounted on a force transducer at the bottom, $7.75 \mathrm{~m}$ from the wave makers and $6.75 \mathrm{~m}$ from the beginning of the slope. The investigated sea states from this data set were also unidirectional. Linear wave generation theory was used to form the paddle movement signal for the piston type wave makers. A schematic drawing of the test layout is shown in figure 2.

\subsection{Data analysis}

The parameters of the selected sea states are shown in table 1. Sea states 1 and 2 were conducted in the DeRisk project on a flat bed while the sea states 3 to 8 were conducted in the Wave Loads project on a sloped bed. Due to the 


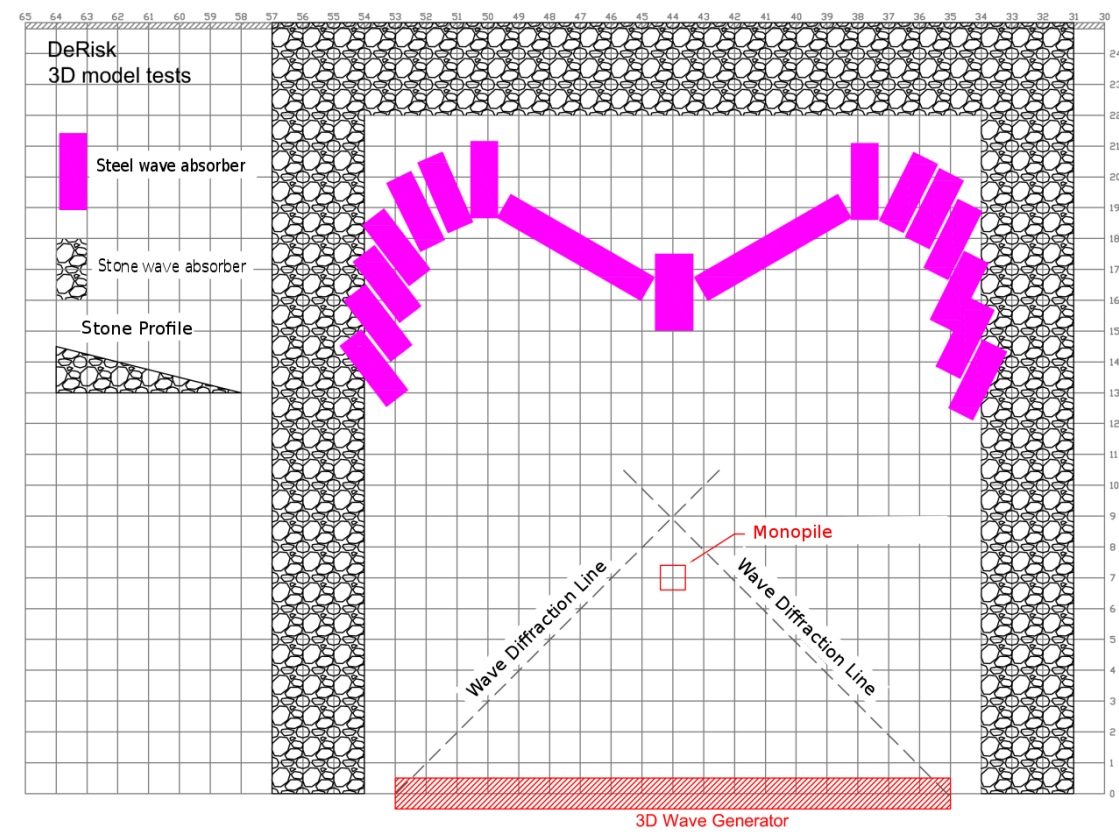

Figure 1: Schematic drawing of the test layout of the DeRisk DHI tests on flat bed [22].

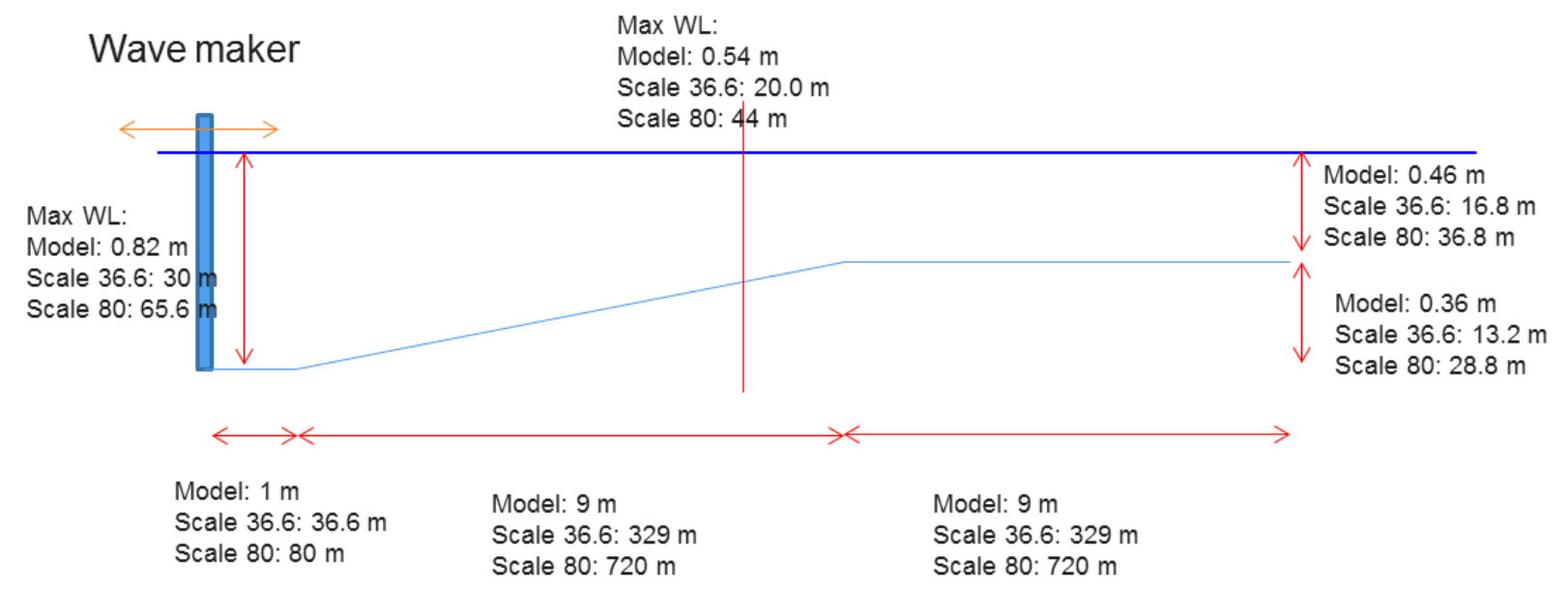

Figure 2: Side view of the test layout used in the Wave Loads project, sloped bed [20].

difference in parameters, the tests in the two test campaigns could not be compared directly. Hence, the first two sea states are used only for validation of the numerical method. The Ursell number [24]

$$
\mathrm{Ur}=\frac{H_{s} L_{p}^{2}}{h^{3}}
$$

was calculated for each sea state to give a measure of nonlinearity and is presented in table 1 . Here $L_{p}$ is the wave length calculated from the linear wave dispersion relation at the peak frequency.

In the analysis of the experimental data, reflections from the artificial beach was observed. Hence, reflection analysis was performed to choose only the sea states with the least reflection affecting the data. The free surface 
elevation time series from 4 different wave gauges at different distances from the wave maker were used to calculate the incident wave and the reflected wave content using the least square method based on the work of Goda and Suzuki [25] and Bredmose et al. [26]. The calculated reflection coefficients,

$$
\operatorname{RefCo}=\frac{\sigma_{\eta_{\text {incident }}}}{\sigma_{\eta_{\text {reflected }}}}
$$

are shown in table 1 , where $\sigma$ is the standard deviation of the time series. The reflection coefficients vary from $7 \%$ to $18 \%$ which might affect the quality of the measured $\eta$ and force time series. However, the main part of the paper results is based on pure numerical calculations and is thus not affected. The equivalent flat bed paddle signal coefficient, EqCo, is also presented for the cases that were used in the investigation of the effect of the bed slope. This parameter is further explained in Section 5.

Figure 3 shows the wave diagram for the selected sea states in addition to the breaking criterion proposed by Goda [27] as

$$
\frac{H_{b}}{g T_{p}^{2}}=A\left(1-\exp \left(-1.5 \pi \frac{h}{L_{p}}\right)\right) \frac{\tanh (k h)}{2 \pi},
$$

where $H_{b}$ is the breaking wave height, $T_{p}$ and $L_{p}$ are the peak wave period and the peak wave length assuming a linear dispersion and constant water depth. The breaking limit is shown in this plot for both $A=0.12$ and $A=0.18$. The sea states were nonlinear but still, for a good part of the wave population, non-breaking. This is beneficial because the numerical model used in this study is a potential flow model as explained in Section 3 and does not include a physics based breaking model.

Table 1: Characteristics of the investigated sea states (Full scale values). Water depth is given at the location of the monopile (MP) and the wave makers (WM)

\begin{tabular}{lllllllllllll}
\hline $\begin{array}{c}\text { Sea } \\
\text { state }\end{array}$ & $\begin{array}{c}\mathrm{h} \\
\mathrm{MP}[\mathrm{m}]\end{array}$ & $\begin{array}{c}\mathrm{h} \\
\mathrm{WM}[\mathrm{m}]\end{array}$ & $\begin{array}{c}k_{p} \mathrm{~h} \\
\mathrm{MP}\end{array}$ & $\begin{array}{c}k_{p} \mathrm{~h} \\
\mathrm{WM}\end{array}$ & $\begin{array}{c}H_{s} \\
{[\mathrm{~m}]}\end{array}$ & $\begin{array}{c}T_{p} \\
{[\mathrm{~s}]}\end{array}$ & $\begin{array}{c}\mathrm{D} \\
{[\mathrm{m}]}\end{array}$ & Slope & Scale & Ur & RefCo & EqCo \\
\hline 1 & 33 & 33 & 0.85 & 0.85 & 7.5 & 15 & 7 & 0 & 50 & 12.3 & $8 \%$ & - \\
2 & 20 & 20 & 1.19 & 1.19 & 5.8 & 9 & 7 & 0 & 50 & 8.0 & $7 \%$ & - \\
3 & 40.8 & 62.4 & 1.23 & 1.68 & 8.3 & 12.6 & 6 & $1: 25$ & 80 & 5.9 & $8 \%$ & 0.9788 \\
4 & 40.8 & 62.4 & 1.06 & 1.43 & 11 & 14 & 6 & $1: 25$ & 80 & 10.9 & $8 \%$ & 0.9967 \\
5 & 30.8 & 52.4 & 1.01 & 1.47 & 8.3 & 12.6 & 6 & $1: 25$ & 80 & 11.6 & $9 \%$ & 0.9978 \\
6 & 30.8 & 52.4 & 0.89 & 1.26 & 11 & 14 & 6 & $1: 25$ & 80 & 18.4 & $10 \%$ & 0.9840 \\
7 & 20.8 & 42.4 & 0.80 & 1.25 & 8.3 & 12.4 & 6 & $1: 25$ & 80 & 22.8 & $18 \%$ & 0.9840 \\
8 & 20.8 & 42.4 & 0.70 & 1.08 & 11 & 14 & 6 & $1: 25$ & 80 & 30.8 & $15 \%$ & 1.3550 \\
\hline
\end{tabular}

The individual waves were separated in the measured time series of the free surface elevation $(\eta)$ with the zero down-crossing technique. The wave height and maximum inline force for each wave was then stored. These data were used to calculate the exceedance probability for each wave based on peak inline force and the crest height based on the individual wave events. For a given maximum inline force, 9 waves with the closest maximum inline force were selected and ensemble averaged to obtain the expected shape of the wave in the specific sea state with the given maximum inline force. This method was initially proposed by Grice et al. [28] for use with free surface elevations and coined as the "designer wave". Later this method was used for inline force time series by Schløer et al. [17] and Ghadirian et al. [18]. Although more than nine wave episodes for the averaged events is desirable, the nature of the extreme events, especially towards the tail of the distribution makes the number of observed peaks close to the largest forces limited. An example of such waves can be seen in figure 5 as gray lines with error bars. The given maximum inline force values are called the target forces hereafter. They were selected from the statistical distribution of inline peaks for each sea state. The choice of these target forces could be related to the return period of the sea states and Ultimate Limit State in the design process. This step, however, was not carried out in this study. 


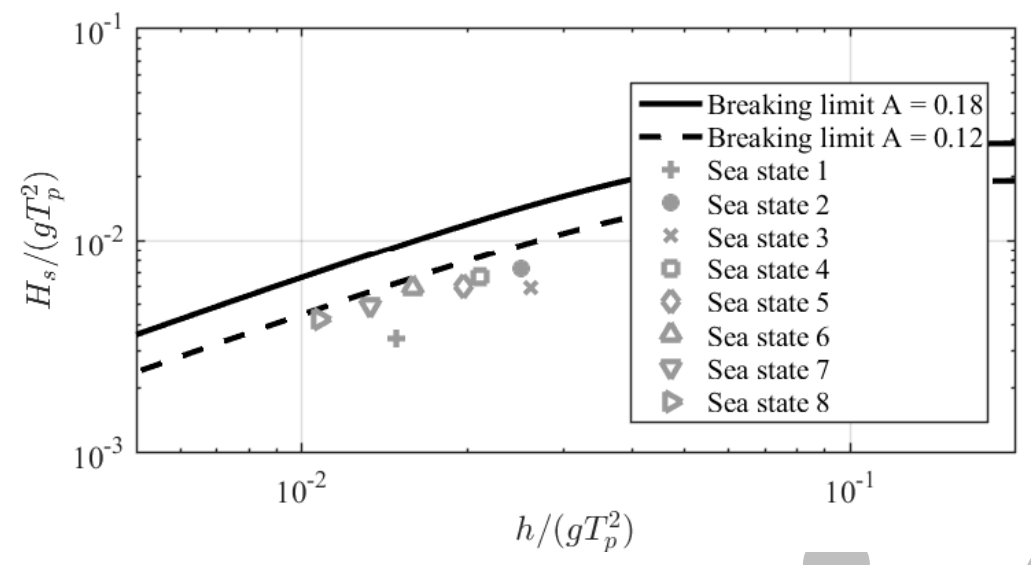

Figure 3: Wave diagram for the investigated sea states.

\section{Wave model and FORM problem}

The numerical method to devise the design wave events and their statistics is a combination of FORM and a fully non-linear wave model. These are described in detail in the following.

\subsection{The numerical flow solver: OceanWave3D}

OceanWave3D [29] is a finite difference potential flow solver that solves the Laplace equation in three dimensions with kinematic and dynamic free surface boundary conditions and kinematic boundary conditions on varying topography. A flux boundary condition on one of the side walls can be used similarly to the wave makers in experiments. This method is linearly consistent with the piston type wave makers to replicate the wave maker motion of the experiments and has been used successfully in previous studies for reproduction of wave tank experiments [30, 31]. A sixth order discretization scheme in space was used for all simulations while the classical explicit four-stage, fourth-order Runge-Kutta scheme was used for time stepping.

A convergence study was performed to make sure that all the waves in each sea state, even with the highest frequency and smallest wave length are well resolved. In all the cases the size of the cells in the wave propagation direction was $2 \mathrm{~cm}$, that is between 120 and 240 points per wave length, while 15 points were used in the vertical direction with clustering of the points towards the free surface. A relaxation zone was used to damp the waves at the end of the numerical flume. The length of this zone was tested to ensure proper damping of the longest waves in all the investigated sea states. The OceanWave3D domain is shown in Figure 4.
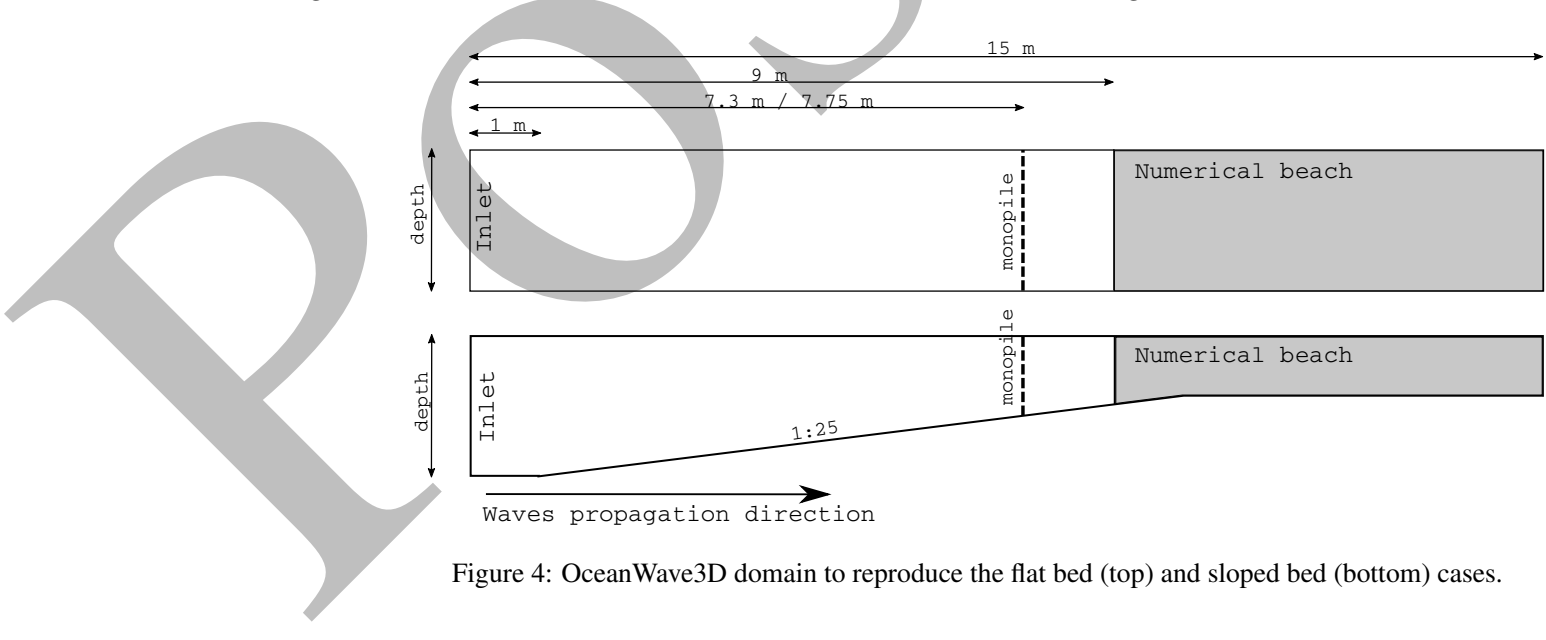

Figure 4: OceanWave3D domain to reproduce the flat bed (top) and sloped bed (bottom) cases. 
To calculate the forces from the kinematics the drag and inertia coefficient must be known. To find the optimum $C_{d}$ and $C_{M}$ for all the sea states, firstly the measured free surface elevation was used to calculate wave kinematics time series at the location of the monopile. Linear wave theory and Wheeler stretching were used for the calculation of the wave kinematics from the free surface elevation. Afterwards the linear least square method was used to calculate the optimum $C_{d}$ and $C_{M}$ which would result in a computed inline force time series closest to the measured force time series. We could not use OceanWave3D simulations for this process because the paddle signal from the experiments were not available and thus did not allow a complete deterministic reproduction of the measured wave field. Another method for the calculation of the force coefficients which was investigated was to calculate the kinematics of the waves at still water level for the three hour random realizations of the sea states, then using the Keulegan-Carpenter and Reynolds number to read the force coefficients from existing experimental tables. However, insignificant differences in the inertia coefficient was observed by comparing them to the initial method. Larger differences were observed between the calculated drag coefficients from the two methods. Since the flow in the cases in this study were strongly inertia dominated it was anticipated that the difference in the drag coefficient would not effect the final conclusions significantly.

\subsection{First Order Reliability Method}

The First Order Reliability Method is used to calculate the most probable wave generation signal to make a wave that creates a target value of maximum inline force $\left(F_{\text {target }}\right)$. The linear wave generation theory [21] is used to create the wave generation signal where

$$
\begin{aligned}
& \sigma_{j}=\sqrt{S_{j} d f} \\
& A_{j}=\sigma_{j} \sqrt{a_{j}^{2}+b_{j}^{2}} \\
& C_{j}=\frac{-2+2 \cosh \left(2 k_{j} h\right)}{2 k_{i} h+\sinh \left(2 k_{j} h\right)} \\
& a p_{j}=\frac{A_{j}}{C_{j}} \\
& \phi_{j}=\arctan \left(\frac{-b_{j}}{a_{j}}\right) \\
& u_{\text {paddle }}(t)=\sum_{j} a p_{j} \omega_{j} \cos \left(\omega_{j} t+\phi_{j}\right) .
\end{aligned}
$$

Here subscript $j$ indicates the element number of the vectors for each frequency. The JONSWAP power spectral density of the free surface elevation is shown by $S$, calculated for a given sea state in Table 1 . The frequency increment is given by $d f$. Angular velocity is shown by $\omega$ and the wave number by $k$. The wave generation signal is shown by $u_{\text {paddle }}(t)$ which is the velocity time series of the wave maker paddles, used as a flux boundary condition in the OceanWave3D simulation. Finally $a_{j}$ and $b_{j}$ are normal distributed variables chosen according to

$$
\left(a_{j}, b_{j}\right) \in \mathcal{N}\left(0, \sqrt{S_{j} \Delta f}\right) .
$$

From the OceanWave3D simulation, the kinematics in the location of the cylinder is obtained. The Rainey formulation of the Morison equations [32] was used to calculate the inline force on the monopile from the kinematics by

$$
\begin{aligned}
F_{O C W 3 D}= & \int_{-h}^{\eta}\left(\rho A C_{M} \frac{D u}{D t}+\rho A C_{m} \frac{\partial w}{\partial z} u+\frac{1}{2} \rho D C_{d} u|u|\right) d z \\
& -\left.\frac{1}{2} \rho A C_{m} \frac{\partial \eta}{\partial x} u^{2}\right|_{z=\eta} .
\end{aligned}
$$

Here $\frac{D u}{D t}=\frac{\partial u}{\partial t}+u \frac{\partial u}{\partial x}+w \frac{\partial u}{\partial z}$ is the material time derivative of the velocity in the wave propagation direction. In (6), $\rho$ is the water density, $A$ is the cross section area of the cylinder, $C_{m}$ is the added mass coefficient, $C_{M}=C_{m}+1$ is the inertia coefficient, $D$ is the cylinder diameter and $C_{d}$ is the drag coefficient. For any set of $\{\underline{a}, \underline{b}\}$, a wave paddle signal for OCW3D was computed. Then an OceanWave3D simulation was run to obtain the free surface elevation and kinematics at the location of the structure.

To calculate the most probable wave generation signal is to find the most probable combination of the random set of numbers $\underline{\alpha}=(\underline{a}, \underline{b})$. Since the random numbers in $\underline{\alpha}$ belong to independent normal distributions, the FORM 
problem can be rewritten as an optimization problem to minimize the summation of square of the random variables, $\underline{\alpha} \cdot \underline{\alpha}^{T}$, when the calculated inline force from the set of random numbers is equal to a target force.

$$
\begin{aligned}
& \min \underline{\alpha} \cdot \underline{\alpha}^{T} \\
& \text { subject to } F_{\text {target }}-F_{O C W 3 D}=0 \\
& \text { where } \underline{\alpha}=\{\underline{a}, \underline{b}\}
\end{aligned}
$$

The optimization problem in (7) was solved using the Sequential Quadratic Programming method [33]. Four different values of $F_{\text {target }}$ were chosen in each sea state, ranging from $0.6 \cdot \rho g h R^{2}$ to $1.7 \cdot \rho g h R^{2}$, where $g$ is the gravitational acceleration, $h$ is the local water depth and $R$ is the radius of the monopile. The chosen values of the normalized inline forces are in the upper range of the analysed experimental results. Values in this range were chosen because the focus of the study is the extreme waves. The final result of the FORM optimization is represented by FORM-OCW3D. Because the variables $\{\underline{a}, \underline{b}\}$ are standard normally distributed around zero, the exceedance probability of the found realization with the given maximum inline force, $F_{\text {target }}$, is given by

$$
\begin{aligned}
& \Phi=e^{\left(\frac{-\beta^{2}}{2}\right)}, \\
& \beta^{2}=\underline{\alpha} \cdot \underline{\alpha}^{T} .
\end{aligned}
$$

This follows from the result that the peaks of a Gaussian process are Rayleigh distributed [34]. Similar method was previously used by Gibson et al. [35] and Jensen [36]. We look at the peak statistics with no extra sorting related to statistical independence.

The only limitation is the present ad hoc breaking filter of the OceanWave3D model, which may require sea state dependent calibration. For all cases of the present investigation a constant threshold of 0.5 was applied.

\section{Validation of the model with measurements}

In this section we present the validation results for the cases on both flat and sloped bed. Time series of the free surface elevation and inline force are presented. The mean relative errors of the FORM results relative to the measurements and the calculated exceedance probabilities are also presented for each case.

\subsection{Flat bed}

\subsubsection{The time series and the relative errors}

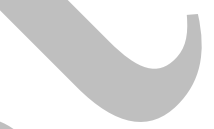

The time series of the ensemble averaged measured free surface elevation and inline force are presented in figure 5 for 9 events with maximum inline force equal to $1 \cdot \rho g h R^{2}$ in sea state 1 . The 9 events are synchronized around the peak force time instant. The error bars in this plot show the standard error of the mean, $\sigma_{N}=\frac{\sigma}{\sqrt{N}}$, where $\sigma$ is the standard deviation and $N$ is the sample size (here 9). The wave appears nonlinear with symmetric trough heights and asymmetric slopes on two sides of its crest. The wave reflection from the monopile is visible as a small wriggle in the following trough of the main wave in the free surface elevation time series. The inline force time series is asymmetric and backwards leaning. A small wriggle in the trough of the inline force time series is noticeable in the measured data which is an indicator of the secondary load cycle [30, 31, 37].

In the same figure the FORM-OCW3D results are presented in blue color. The general behavior is consistent with the measurements. The shape of the wave and the backward leaning of the inline force time series are captured in these numerical results. However, there is a small phase shift between the free surface elevation time series of the measurements and FORM-OCW3D. The small phase shift might come from the inaccuracies in the estimated $C_{d}$ and $C_{M}$. A larger $C_{d}$ value would shift the peak force for large waves to a time instant closer to the wave crest. Another reason for the phase shift might be the higher-order diffraction waves which are not considered in the model. In addition, the secondary load cycle is not visible in FORM-OCW3D as expected since, the structure is not present in the numerical domain and the forces are only calculated using the Morison equation and the Rainey corrections.

The results from FORM application to linear and second-order theory (Dean and Sharma method [21]) are also presented denoted by FORM-F1 and FORM-F2 respectively. The underlying theory and similar results and the theory 
were previously presented in the work by Ghadirian et al. [18]. The linear wave presented in dashed black line is more asymmetric in trough height and in the slopes leading and proceeding the main crest than the measured wave. Moreover, there is a large phase shift between its crest and the crest of the measured wave. The inline force time series of FORM-F1 is symmetric, peaking at the peak time of the measured ensemble averaged time series. It should be noted that the FORM-F1 result is identical to the analytical New Force model introduced by Schøer et al. [17] as detailed in Ghadirian et al. [18]. The FORM-F2 free surface elevation result is more consistent with the measurements than the New Force model wave. There is also a phase shift between the second-order wave crest and the measured wave crest time. The shape of the second-order wave is asymmetric in trough height and in wave slope but generally in better consistency than FORM-F1 with the measured $\eta$. The second-order force time series shows different slopes before and after the peak only slightly better than the New Force results.

Similarly the time series of the ensemble averaged measured free surface elevation and inline force are presented in figure 6 for 9 events with maximum inline force equal to $1.2 \cdot \rho g h R^{2}$ in sea state 2 . The wave appears nonlinear with symmetric trough heights and asymmetric slopes on two sides of its crest. Even though the absolute crest height is smaller than the crest height of the case shown in figure 5 it is larger relative to the water depth and the wave is more nonlinear. The general behavior of the FORM-OCW3D free surface elevation is consistent with the measurements. However, the phase shift between the free surface elevation time series of the measurements and FORM-OCW3D is larger in this case compared to the case shown in figure 5. The consistency of the FORM-F1 and FORM-F2 results with the measurements is similar to the previous case. It was observed that the breaking filter in the numerical domain has been activated and smoothened some of the wave at the location of breaking. Probably because of the activation of the breaking filter the time series of FORM-OCW3D are not as consistent with the measurements as in figure 5. The case shown in figure 6 was the largest target force in sea state 2 . The third target force in the same sea state is shown in figure A.21 which displays a better consistency between the FORM-OCW3D results and the wave averaged measurements.
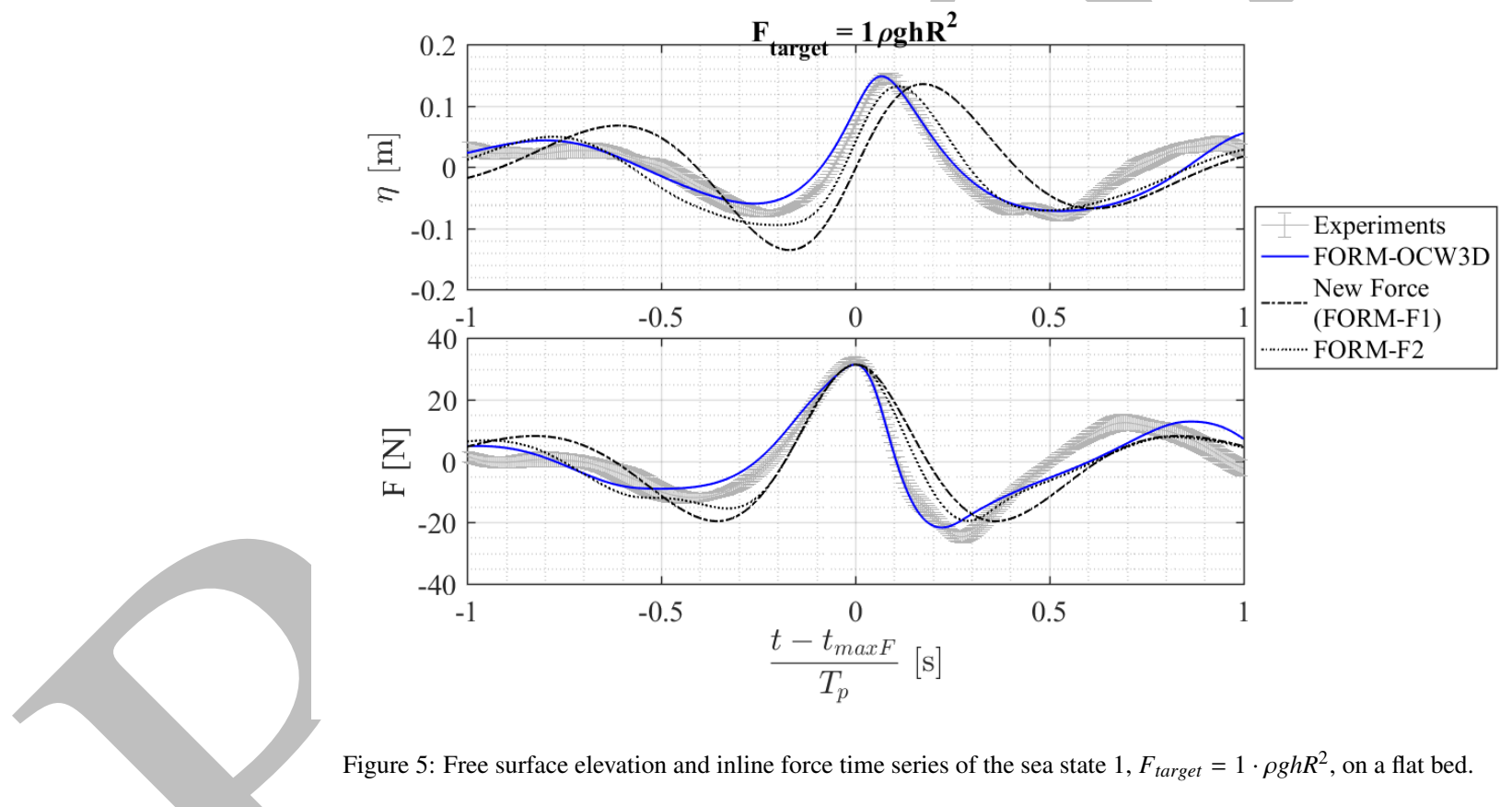

Figure 5: Free surface elevation and inline force time series of the sea state $1, F_{\text {target }}=1 \cdot \rho g h R^{2}$, on a flat bed.

Figure 7 shows the mean relative errors of the inline force and free surface elevation time series between the ensemble averaged measurements and the numerical results namely FORM-F1, FORM-F2 and FORM-OCW3D. The absolute relative phase shift between the free surface elevation time series of the measurements and the numerical results is also presented in this figure relative to the peak period of each sea state. The relative error from $-1.5 \cdot T_{p}$ to 


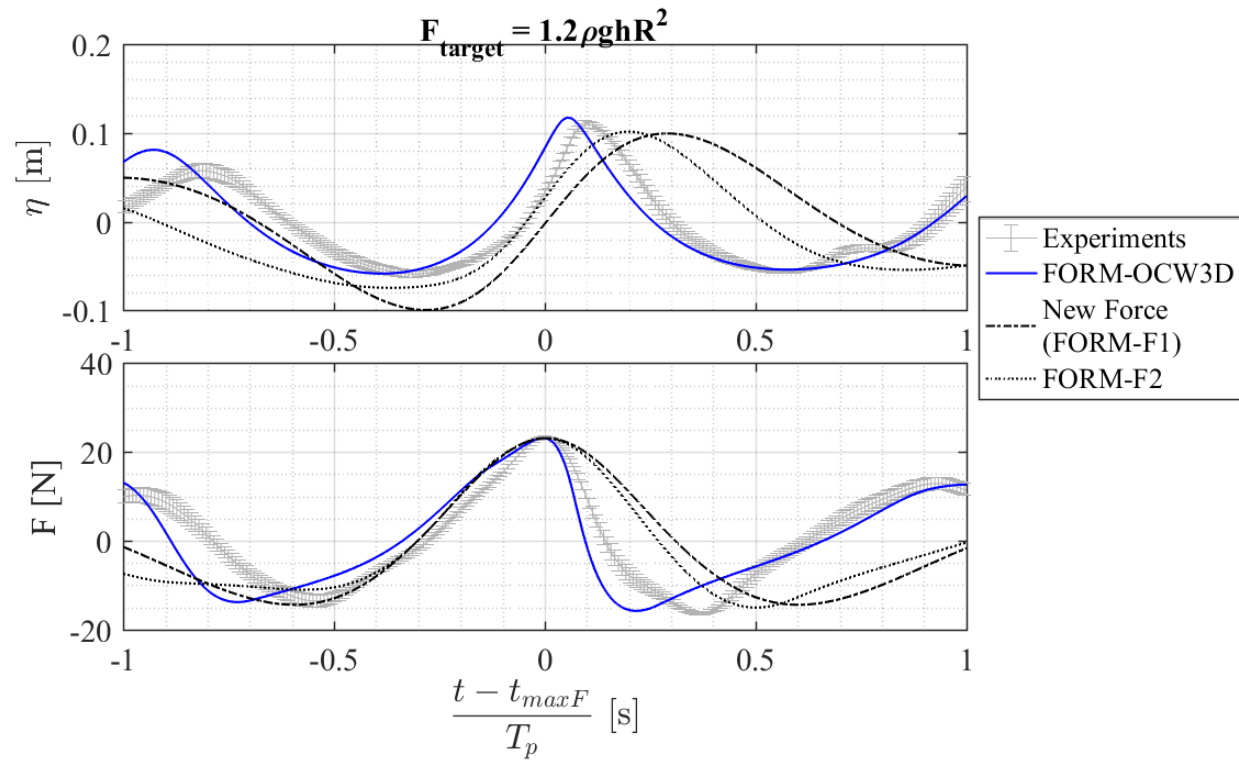

Figure 6: Free surface elevation and inline force time series of the sea state $2, F_{\text {target }}=1.2 \cdot \rho g h R^{2}$, on a flat bed.

$1.5 \cdot T_{p}$ around the peak force time is calculated by

$$
E=\frac{\sqrt{\overline{\left(\mathbf{X}-\mathbf{X}_{\text {measured }}\right)^{2}}}}{\max \left(\mathbf{X}_{\text {measured }}\right)},
$$

where $\mathbf{X}$ can be the inline force or free surface elevation for each time step.

It is observed that the relative errors of the free surface elevation of FORM-OCW3D from $-1.5 \cdot T_{p}$ to $1.5 \cdot T_{p}$ non-dimensional time does not exceed $16 \%$ and its average is around $12 \%$. The mean relative error for the inline force of FORM-OCW3D time series is smaller than $19 \%$ with an average around $10 \%$ for all the cases. One probable reason for the inconsistencies might be the fact that the model does not include higher-order diffraction. Another probable reason, linked to this limitation, is the secondary load cycle in the wave averaged measured inline force history which does not appear in the numerical results. The relative phase shift between the $\eta$ time series of FORM-OCW3D and the measurements is smaller than $6 \%$ for all the cases as shown in the bottom plot of this figure.

The relative errors for $\eta$ and $F$ for FORM-F1 and FORM-F2 are also shown in figure 7. The relative errors of FORM-F1 results are significantly larger than the ones from FORM-OCW3D. The relative errors of FORM-F2 results are always smaller than FORM-F1 as expected. The errors of FORM-F2 are larger than the FORM-OCW3D results for all cases except the largest target force 1.2. $\rho g h R^{2}$ in sea state 2 . The reason might be activation of the breaking filter in the OceanWave3D model in this case. For all the sea states the mean relative error of the free surface elevation is around 27\% for FORM-F1 and 15\% for FORM-F2 in average. In average of all the cases, the mean relative error of the inline force for FORM-F1 and FORM-F2 is $18 \%$ and $14 \%$ respectively. From figure 7 , it is observed that implementing the FORM-OCW3D model has reduced the errors around 40\% relative to $\eta$ of FORM-F2 and $80 \%$ relative to F of FORM-F2.

\subsubsection{The probability plots}

Figure 8 shows exceedance probability plots of the force peaks and crest heights of random realizations from sea state 1 and 2. The measurements are shown in gray lines with error bars. These data were extracted from 3 hour (full scale) random realizations for each sea state. After the waves were separated using zero down-crossing 20 sets of wave event collections were randomly selected from them with the same size as the original set of waves. Same size of the samples means that in each of the 20 sets there might be repeated wave events. This process is called bootstrapping with replacement [38] and serves to estimate the stochastic variability for the obtained exceedance probability curve 

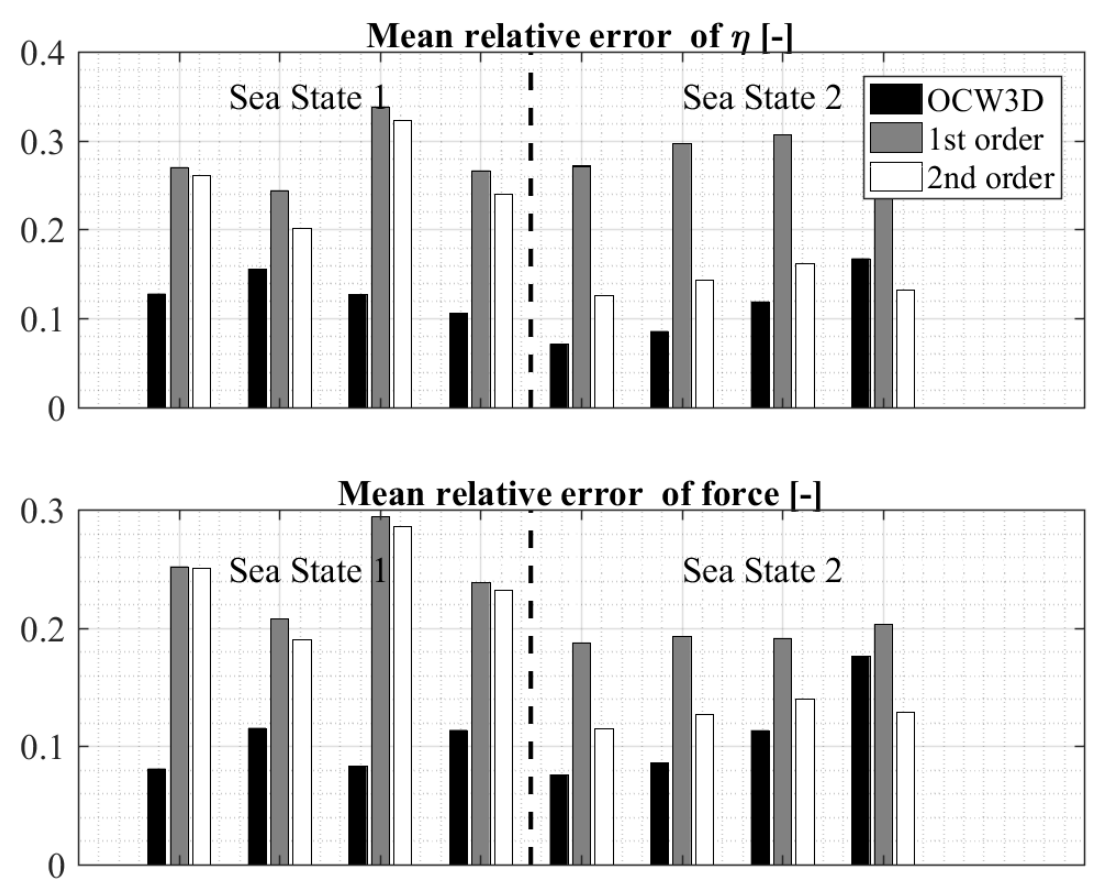

Relative phase shift between $\eta$ time series [-]
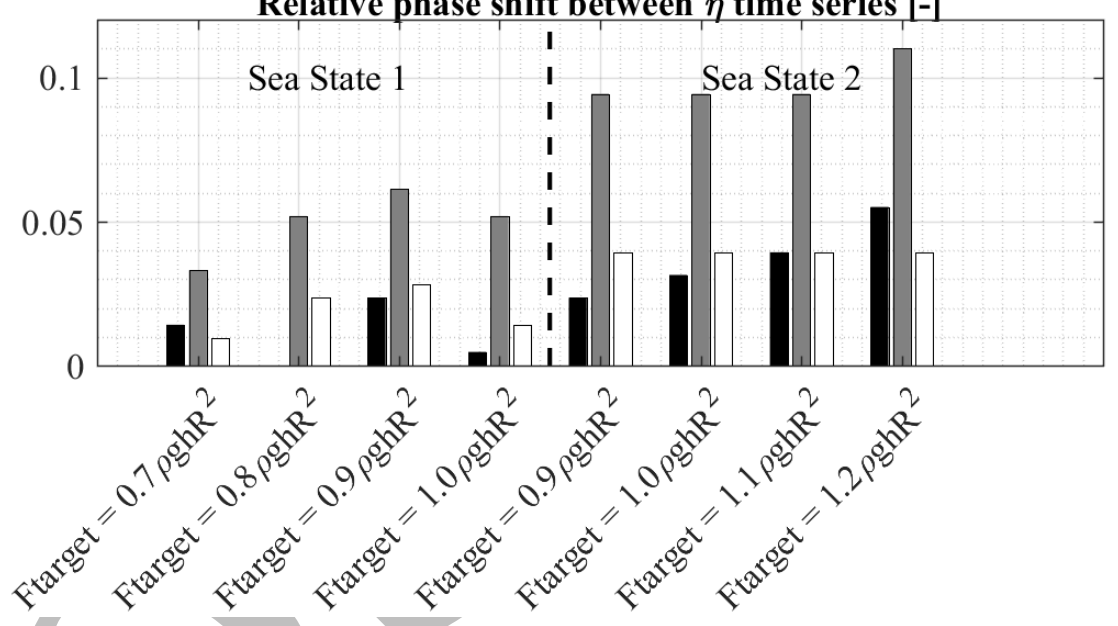

Figure 7: Mean relative error of the free surface elevation and inline force time series between the ensemble averaged measurements and the numerical results for sea states 1 and 2 on flat bed.

of a single realization. The advantage of this method is to avoid performing 20 random realization simulations or experiments for the same sea state.

A random realization, with an independent stochastic seed, of each sea state was also simulated in OceanWave3D and the results are shown in black curves in the plots in figure 8. For both sea states the OceanWave3D statistical distributions of maximum inline force and maximum crest heights are in relative good agreement with the measurements for high probabilities of exceedance but they deviate in the lower exceedance probabilities. This can be explained by stochastic variability and also to a lesser extent the limits of OceanWave3D which does not include a physics based breaking model.

The estimation of the exceedance probability from the FORM problem is also presented in the exceedance probability plots of inline force with blue filled circles. We expect these results to be in the range of the variations of 
the random realizations of the OceanWave3D simulations. A good consistency between the FORM results and the random realizations is thus observed except for the largest FORM force of sea state 2. It is worth mentioning that the case with the largest target inline force in the second sea state, the right side plot, seems to be in-converged, since the calculated exceedance probability of this case from the FORM problem is much lower than the random realization from the OceanWave3D simulations. However, since these cases are only for validation the re-computation of this case is omitted.
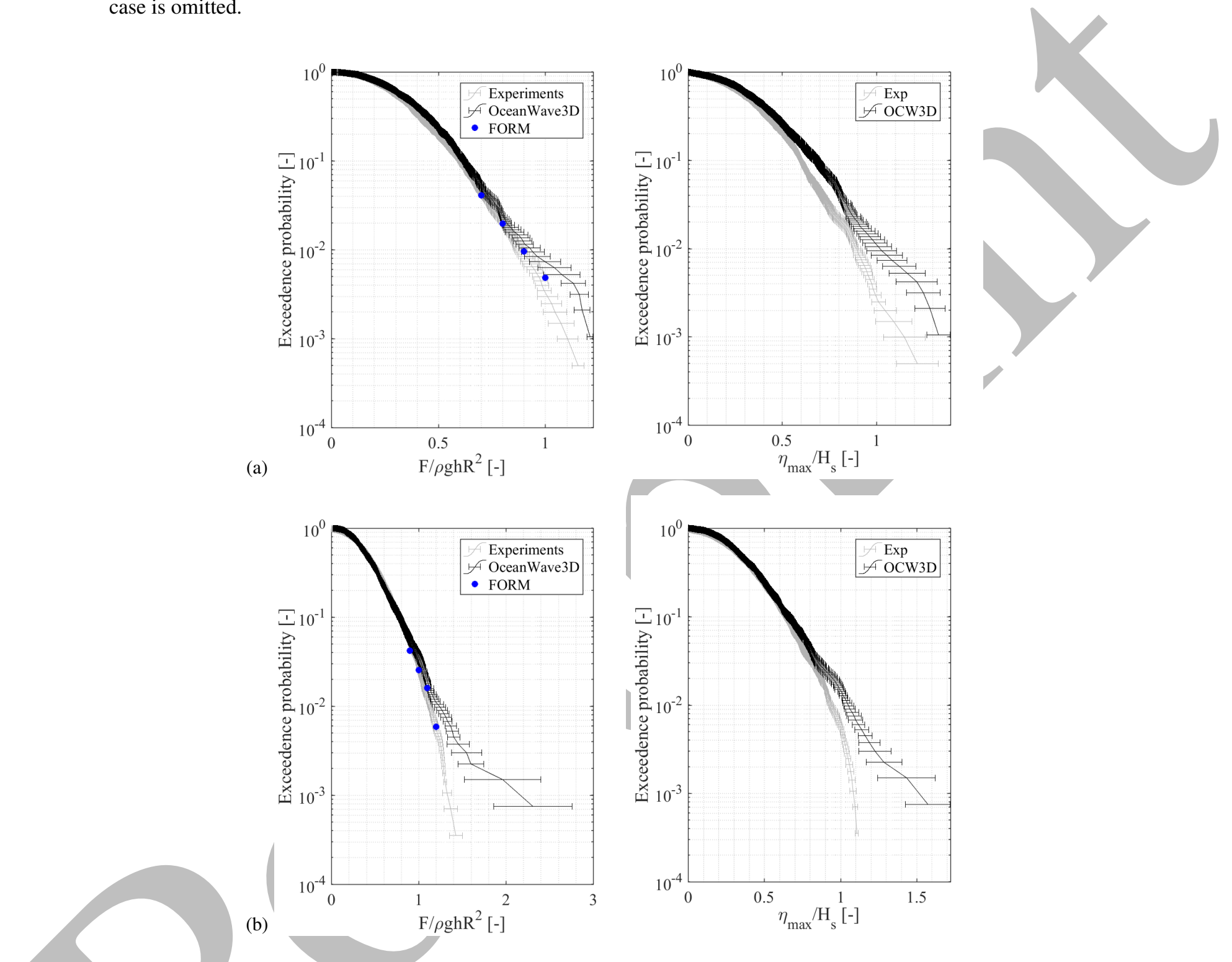

Figure 8: Exceedance probabilities relative to maximum inline force and maximum crest height from random realization tests, numerical simulations in OceanWave3D and numerical results from FORM problem for two sea states. (a) sea state 1 and (b) seat state 2.

\subsection{Sloped bed}

\subsubsection{The time series and the relative errors}

The time series of the ensemble averaged measured free surface elevation and inline force are presented in figure 9 for 9 waves with maximum inline force as large as $1 \cdot \rho g h R^{2}$ in sea state 3 . The wave is nonlinear with an asymmetric shape. The general behaviour of the FORM results is very close to the ensemble averaged measurements of the free 
surface elevation and inline force time series. The shape of the wave and the backward leaning of the inline force time series are captured. The initial measured water level could not be determined accurately. For this reason in the comparison of the free surface elevations a zero mean has been imposed in both experiments and numerical results. After this correction, a good consistency between the measurements and the numerical results can be observed in this figure.

Similar time series of the ensemble averaged measured free surface elevation and inline force are presented in figures A.23 to A.25 in the appendix for sea states 5 to 8 with largest maximum inline force in each case. Similar consistency between the FORM results and the wave averaged measurements is observed for all the cases. However, in sea states 6 to 8 a larger preceeding slope is observed in the free surface elevation time series of the FORM results. In general the FORM results are more skewed in these cases than the measurements.

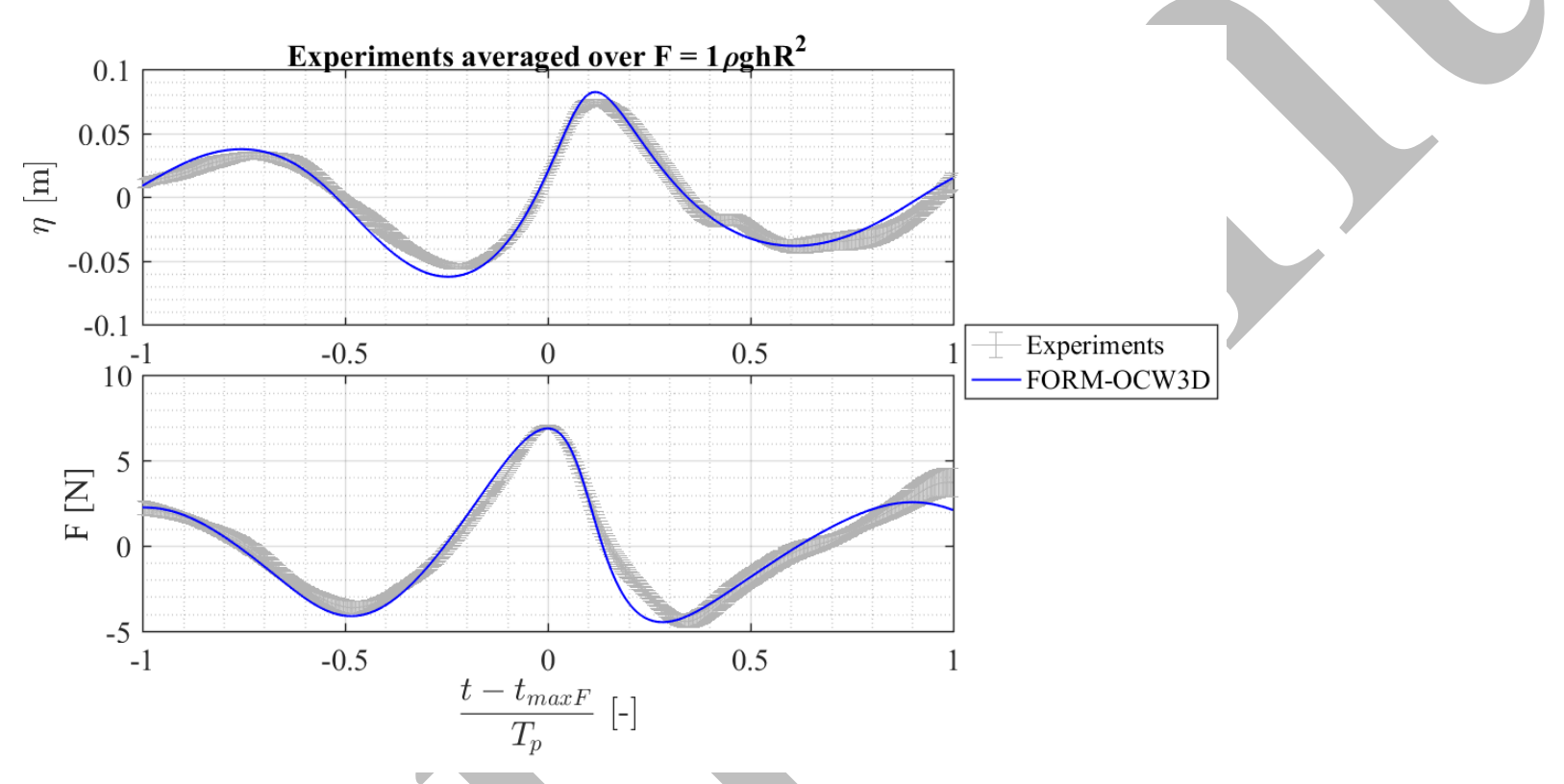

Figure 9: Free surface elevation and inline force time series of the sea state $3, F_{\text {target }}=1 \cdot \rho g h R^{2}$, on a sloped bed.

Figure 10 shows the mean relative errors of the inline force and free surface elevation time series between the ensemble averaged measurements and numerical results for all the cases on a sloped bed, sea states 3 to 8 . The absolute relative phase shift between the free surface elevation time series of the measurements and numerical results is also presented in this plot relative to the peak period of each sea state. It is observed that for all the sea states, the average of the mean relative error for the inline force time series is around $8 \%$. The relative errors of the free surface elevation from the leading zero down-crossing to the following zero down-crossing does not exceed $12 \%$ in all cases with average mean relative error around $8.6 \%$. The phase shift between the free surface elevation time series does not exceed $3 \%$. In general, a good consistency between the numerical results and the measurements is observed in the cases on sloped bed.

\subsubsection{The probability plots}

Figure 11 shows the exceedance probabilities of maximum inline forces and maximum crest heights for sea states 3 to 8 . The measurements are shown in gray lines with error bars. As the maximum inline force increases the probability of the exceedance decreases. The black curves show the same general behavior for the results from a three hour (full scale) random realization of each sea state simulated in OceanWave3D. In this figure we can see that the statistical distribution of the maximum inline forces are generally consistent between the measurements and the OceanWave3D simulations.

The estimation of the exceedance probability from the FORM problem is presented with filled blue circles. It is expected that these results lie in the range of the variations of the random realization of the OceanWave $3 \mathrm{D}$ simulations 


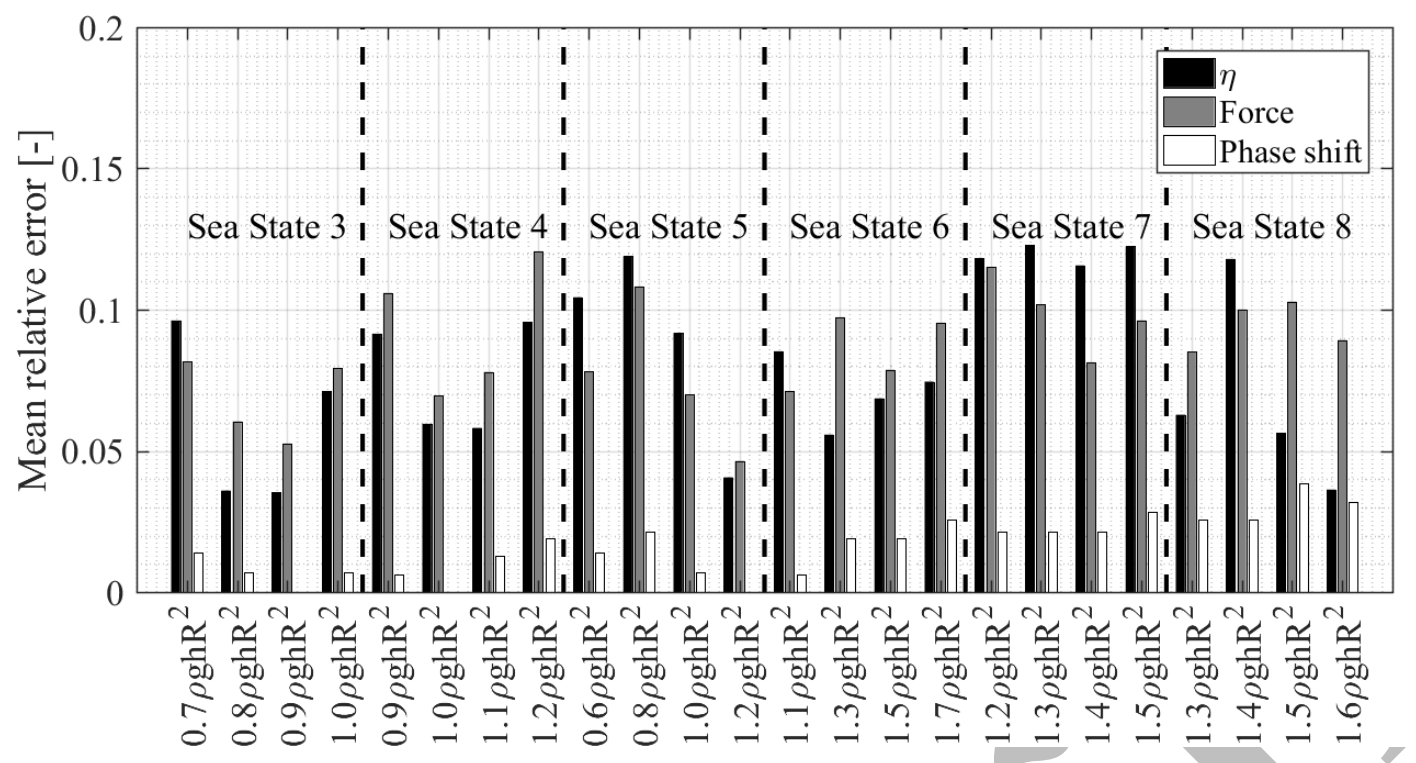

Figure 10: Mean relative error of the free surface elevation and inline force time series between the ensemble averaged measurements and the numerical results for sea states 3 to 8 on sloped bed.

and so in the range of the variation of the measurements. Similar plots for sea states 4 to 8 are shown in the appendix figures A.27 to A.31 and a good consistency between the FORM results and the random realizations is observed for all 6 sea states.
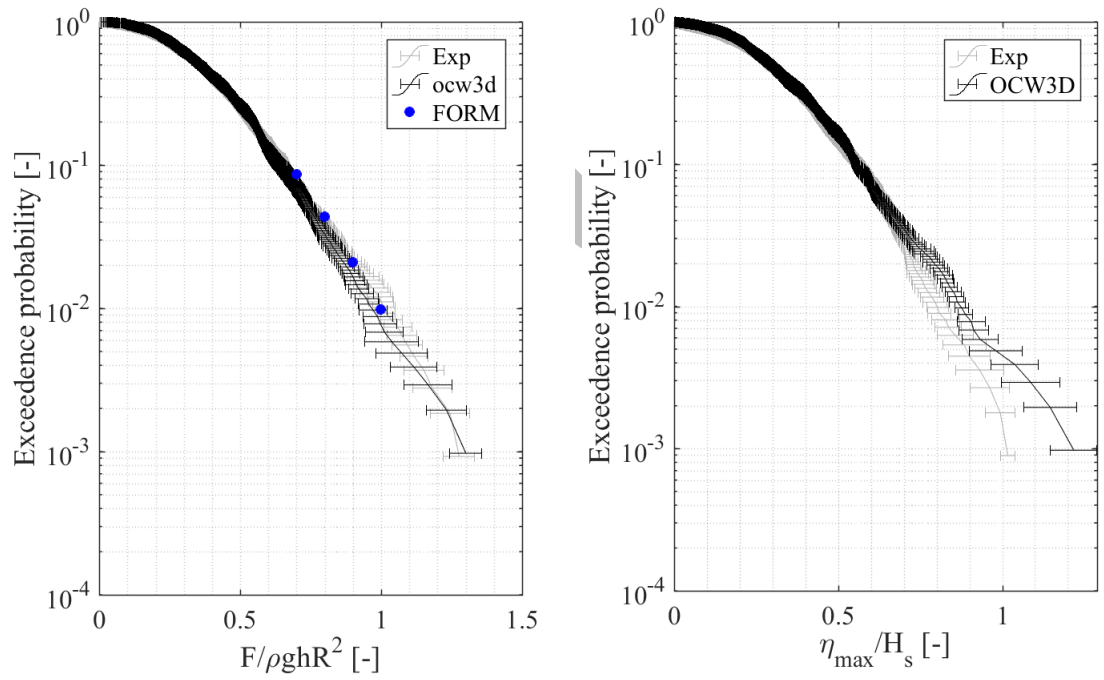

Figure 11: Exceedance probabilities relative to maximum inline force from random realization tests, numerical simulations in OceanWave3D and numerical results from FORM probem for sea states of 3 on sloped bed.

The results in this section show that the method is valid to use for similar cases on a sloped bed. In the next section the application of the method for investigation of the effect of the bed slope is presented. 


\section{The effect of the bed slope}

The main aim of the current study is to investigate the effect of the bed slope on the force statistical distribution and time history. To achieve this an equivalent numerical domain with flat bed and the same depth at the location of the cylinder was created. A series of calibration simulations were performed to obtain the same sea state parameters,

$$
H_{s} \quad \text { and } \quad T_{1}=\frac{\int S d f}{\int f S d f},
$$

for these simulations on the equivalent flat bed at the location of the cylinder as on the sloped bed simulations. The time scale $T_{1}$, however, is hardly changed over the slope. Then the same target force levels were used for the FORM analysis in both setups. The FORM problem was solved this time around the new OceanWave3D domain for all the cases in the equivalent sea states. The equivalent flat bed paddle signal coefficient was then calculated for the sea states on a flat equivalent bed similarly to the other sea states in Table 1.

It should be mentioned that the sea states 7 and 8 mentioned in Table 1 could not be simulated on the equivalent flat bed in the OceanWave3D domain, therefore the $H_{s}$ was decreased in these two cases by $10 \%$ and $30 \%$ respectively to be able to have random long time realizations of the same sea states on sloped and equivalent flat bed.

\subsection{Comparison of the sloped bed with the equivalent flat bed: the time series and their frequency content}

The free surface and inline force time series and their frequency content are presented in figure 12 to 14 for sloped and equivalent flat bed for sea state 3,5 and 8 with the largest target forces, while the similar results for the sea states 4, 6 and 7 are presented in the appendix figures A.32 to A.34. The results for the sloped bed are shown in gray color. The consistency of these results with the measurements are previously shown in section 4.2. The black curves show the results from the equivalent flat bed numerical domain. It is observed that the force time series are very similar in sea states 3, 4 and 5 but they do show larger differences in sea states 6,7 and 8. The same similarity is visible in the free surface elevation time series. Larger differences around the peak and second harmonic frequencies are observed in the PSD of these time series. With more investigation in all the cases it was observed that the energy content around the double peak frequency is larger in the equivalent flat bed cases. More investigation for the nature of this difference was performed and the results are shown in section 6 .

In the cases from sea states 6,7 and 8 the wave episodes have more skewness on the flat bed than on the sloped bed while the wave heights are smaller on a flat bed. The differences between the force time series are smaller than the differences between the $\eta$ time series. This similarity of the inline force times series between the flat and sloped bed cases is specially clear from trough to trough. The frequency content around the peak frequency is always higher in the sloped bed cases than in the flat bed cases.

\subsection{Exceedance probabilities of maximum inline force and crest height}

Figures 15 to 17 show the exceedance probabilities relative to the non-dimensional inline force peaks (left hand side plot) and crest heights (right hand side plot) for sea states 3,5 and 8. The similar results for the sea states 4,6 and 7 are moved to the appendix figures A.32 to A.34 for brevity. The plots on the right hand side show the agreement of cases with and without a sloped bed. The curves with error bars are obtained from only one realization of the random phase sea state time series using bootstrapping.

On the left hand side plots in addition to the results from the random phase realization the results from FORM simulations are shown for four different target maximum inline force. The differences between the curves with error bars and the FORM results are due to the stochastic uncertainty of the random phase realizations. From these plots, the exceedance probability is always higher for the cases on a sloped bed in all the sea states. This observation is generally in agreement with the full probability curves from the random realizations. The increase of the exceedance probability on a sloped bed is more significant in sea state 7 and 8 . It is worth mentioning that the different exceedance probabilities for the same target forces on sloped bed versus the equivalent flat bed means that the linear combination of the parameters, $\{\underline{a}, \underline{b}\}$, is different at location of the wave paddles in the cases on different slopes.

The ratio between the exceedance probabilities for fixed non-dimensional force value on the sloped bed and flat bed are shown in figure 18. For sea states 3, 4 and 5 an increase in the ratio is observed as the target inline force is increased in each sea state. The ratio changes from 1.05 for a small target force $0.6 \rho g h R^{2}$ in sea state 5 to 1.25 

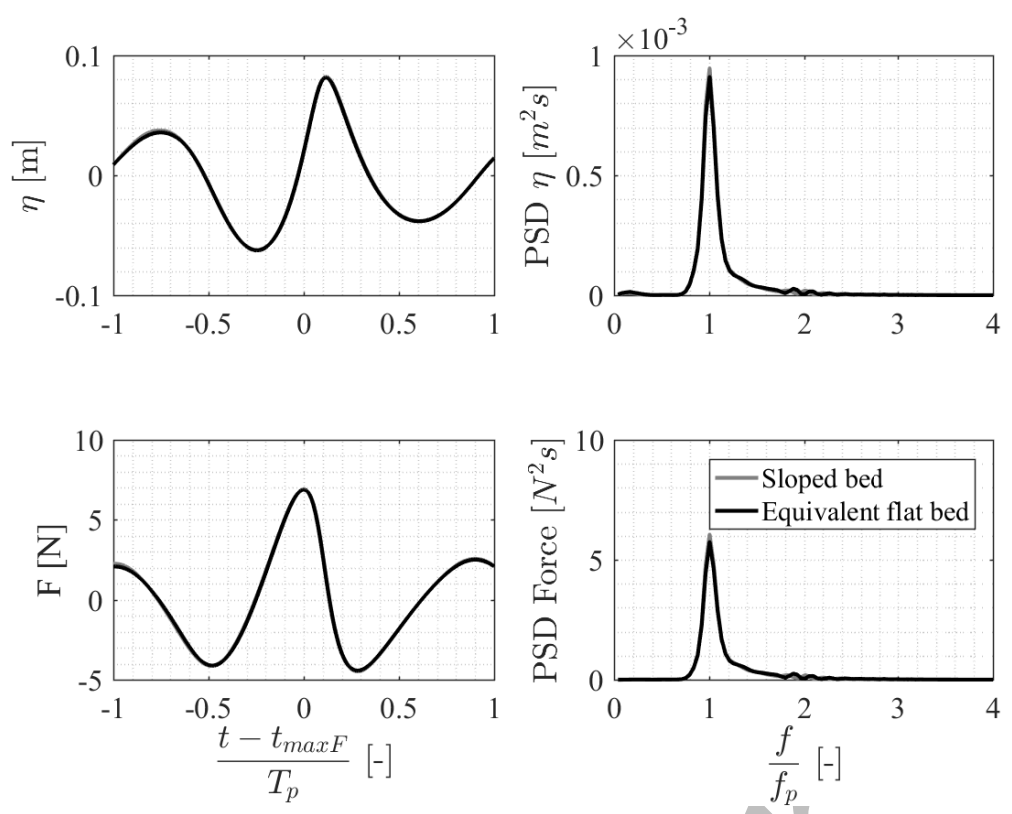

Figure 12: Free surface elevation and inline force time series and power spectral density for numerical results of a case in sea state 3 with $F_{\text {target }}=1 \cdot \rho g h R^{2}$ with sloped bed and the equivalent flat bed.
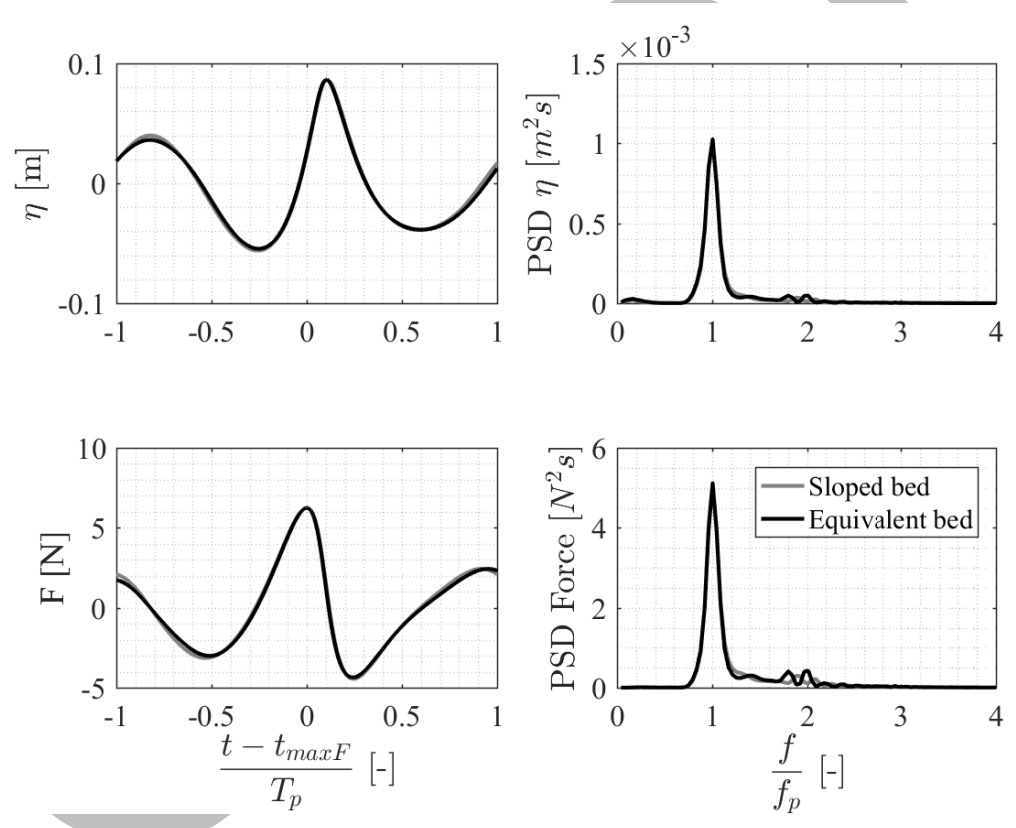

Figure 13: Free surface elevation and inline force time series and power spectral density for numerical results of a case in sea state 5 with $F_{\text {target }}=1.2 \cdot \rho g h R^{2}$ with sloped bed and the equivalent flat bed.

for the largest target force of $1.2 \rho g h R^{2}$ in sea state 5 . The increase in the ratio in each sea state might be explained by the increase of contribution of the longer waves which are effected by the shoaling effect more significantly. The nonlinear content of the force time series are analyzed in the next section.

The ratio of exceedance probabilities in sea state 6,7 and 8 in figure 18 have a different and non-monotonic trend unlike in sea states 3,4 and 5. The ratio is significantly larger and reaches about 2 for these conditions. From Table 1 

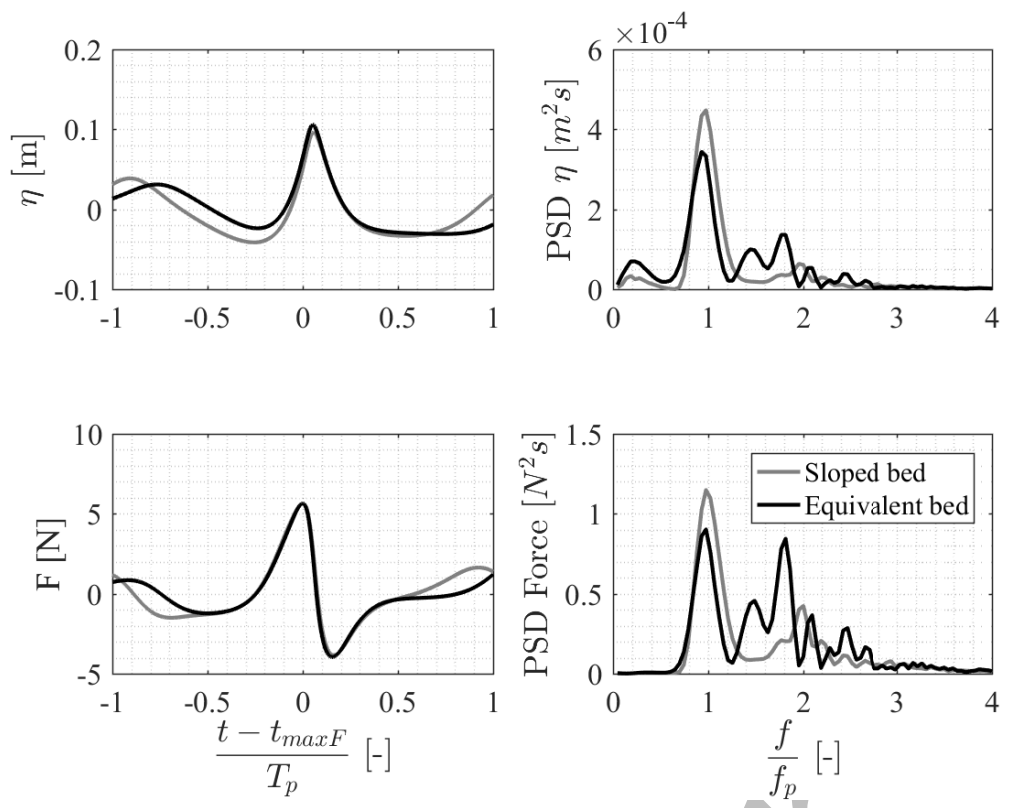

Figure 14: Free surface elevation and inline force time series and power spectral density for numerical results of a case in sea state 8 with $F_{\text {target }}=1.6 \cdot \rho g h R^{2}$ with sloped bed and the equivalent flat bed.
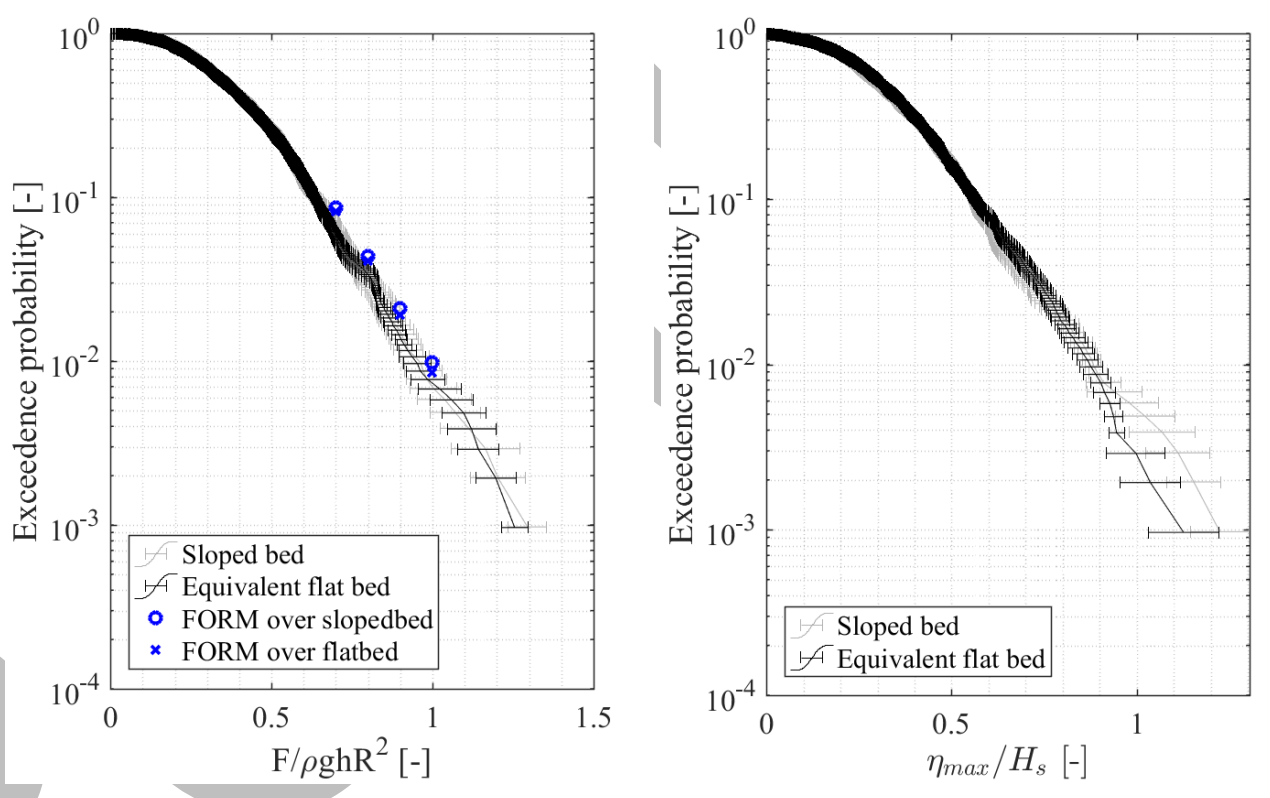

Figure 15: Exceedance probabilities relative to the non-dimensional maximum inline force (left hand side plot) and maximum crest height (right hand side plot) for sea state 3 . The results from FORM calculations are also included in the left hand side plot for sloped bed and equivalent flat sea bed.

it is visible that the three sea states 6, 7 and 8 have much larger Ursell numbers and this increased nonlinearity is expected to cause this dramatic increase in exceedance probability for the sloped cases. The implication for design is dramatic and should be noted. 

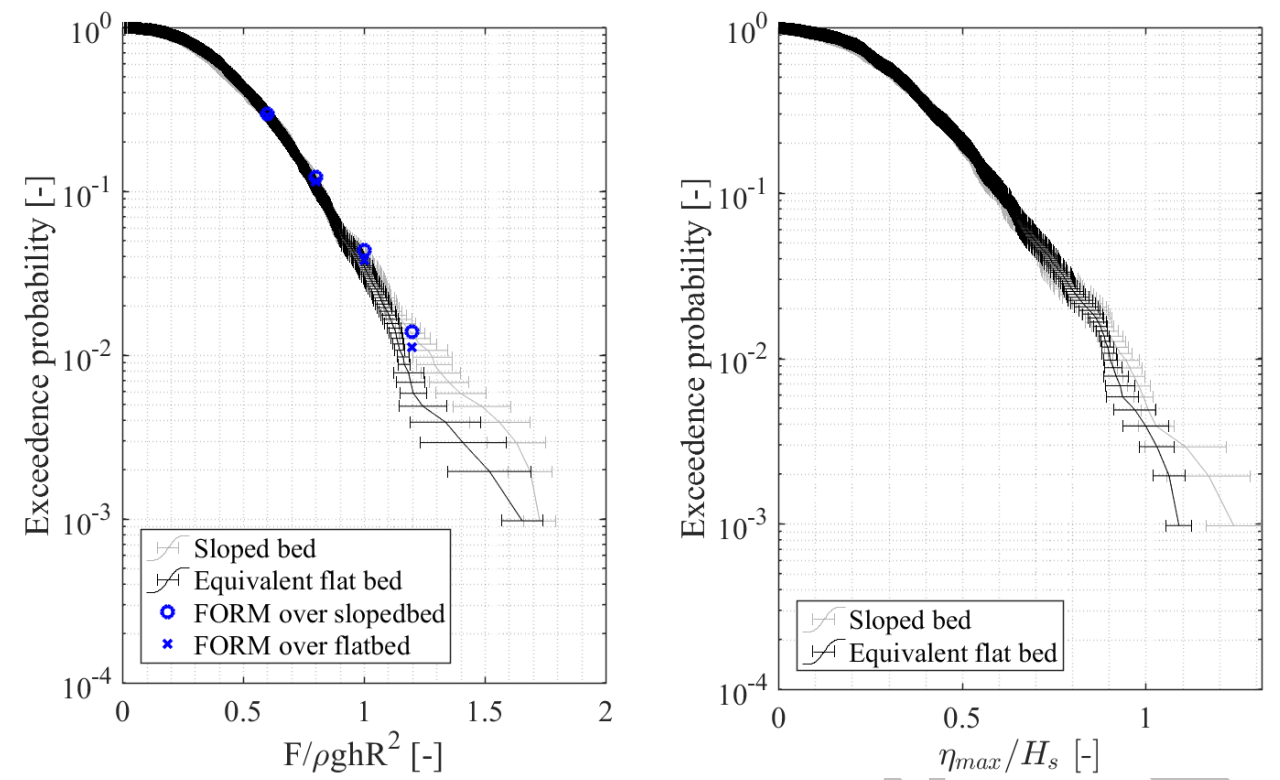

Figure 16: Exceedance probabilities relative to the non-dimensional maximum inline force (left hand side plot) and maximum crest height (right hand side plot) for sea state 5. The results from FORM calculations are also included in the left hand side plot for sloped bed and equivalent flat sea bed.
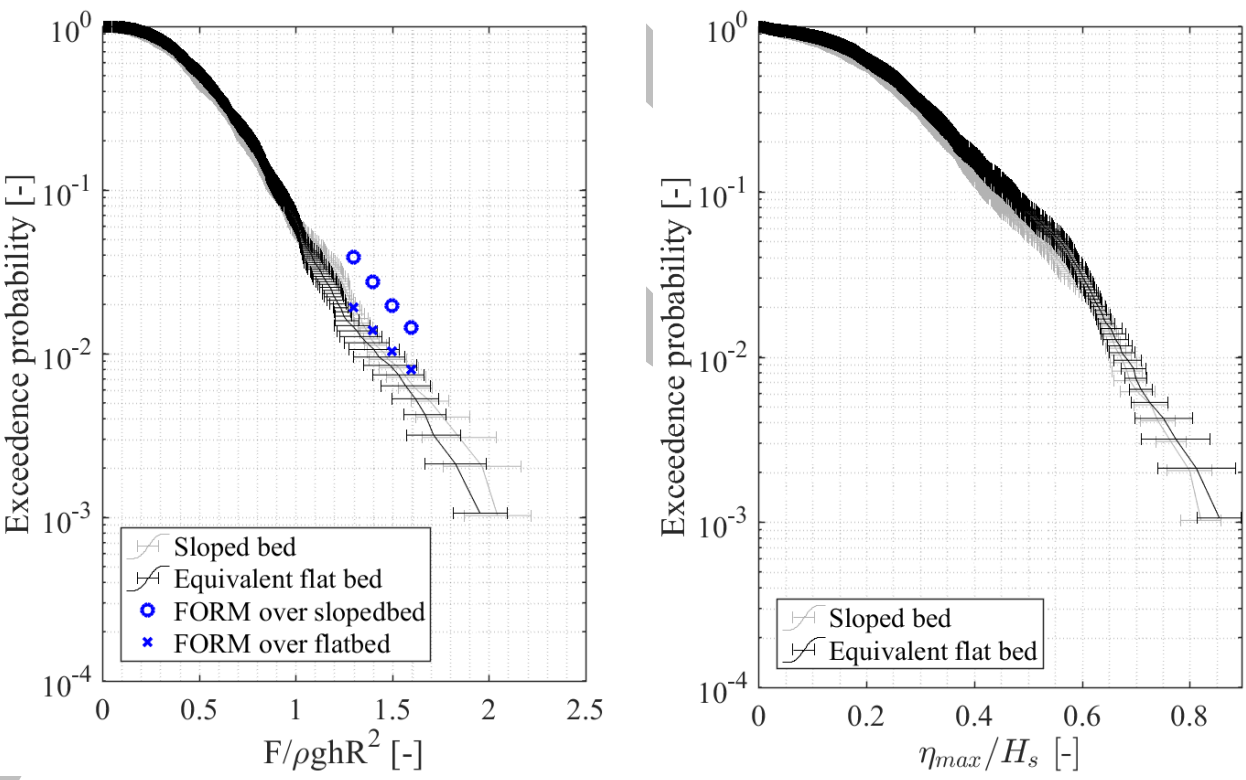

Figure 17: Exceedance probabilities relative to the non-dimensional maximum inline force (left hand side plot) and maximum crest height (right hand side plot) for sea state 8 . The results from FORM calculations are also included in the left hand side plot for sloped bed and equivalent flat sea bed.

\section{Harmonic analysis of force components}

To investigate the effect of the bed slope on the produced force episodes in details, the harmonic separation technique was used based on the work by Fitzgerald et al. [39]. The method is built up on the assumption that 


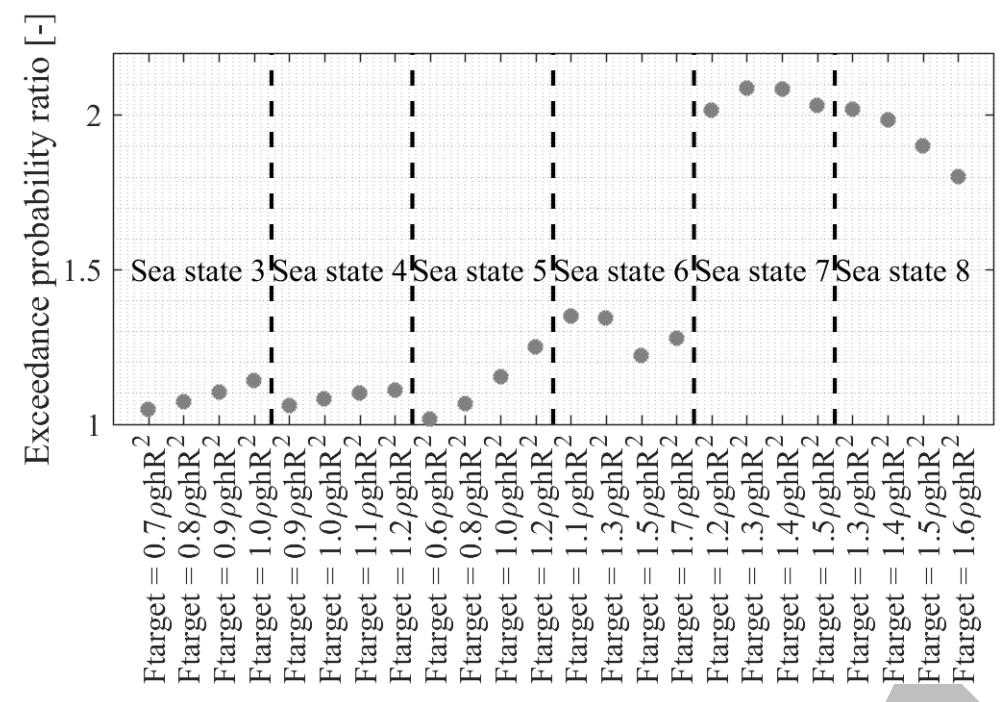

Figure 18: The ratio between the exceedance probabilities of the same load in sloped sea bed and equivalent flat bed.

the viscous effects are negligible relative to the potential flow effects. In addition, the classic Stokes perturbation expansion is used to describe the higher-harmonic nonlinear force structure and devise its decomposition from phase shifted input signals. Thus for a linear force signal of amplitude $A$, frequency $\omega$ and wave number $k$, the following harmonic structure will emerge

$$
F=A f_{11} \cos \phi+A^{2}\left(f_{20}+f_{22} \cos 2 \phi\right)+A^{3}\left(f_{31} \cos \phi+f_{33} \cos 3 \phi\right)+A^{4}\left(f_{42} \cos 2 \phi+f_{44} \cos 4 \phi\right) .
$$

Here the coefficients $f_{m n}$ represent the wave-to-force transfer functions and $\phi$ is the phase of the linear component of the incident wave. Fitzgerald et al. [39] suggested that the harmonic hierarchy of regular Stokes waves can also be used to approximate the phase focused wave groups. In such cases the Stokes terms are replaced with summation of products of frequency components. In the present work we can assume that the solutions to the FORM problem is a highly nonlinear focused wave group. The solutions of the FORM problems can then be used to create paddle signals with $\frac{1}{2} \pi, \pi$ and $\frac{3}{2} \pi$ phase shifts relative to each other. The original force time series are noted by $F_{0}$ and the force time series resulting from the phase shifted paddle signals are denoted by $F_{90}, F_{180}$ and $F_{270}$ respectively. Therefore, different harmonics of the force time series can be divided as

$$
\begin{aligned}
& \frac{\left(F_{0}-F_{90}^{H}-F_{180}+F_{270}^{H}\right)}{4}=A f_{11} \cos \phi+A^{3} f_{31} \cos \phi+O\left(A^{5}\right) \\
& \frac{\left(F_{0}-F_{90}+F_{180}-F_{270}\right)}{4}=A^{2} f_{22} \cos 2 \phi+A^{4} f_{42} \cos 2 \phi+O\left(A^{6}\right) \\
& \frac{\left(F_{0}+F_{90}^{H}-F_{180}-F_{270}^{H}\right)}{4}=A^{3} f_{33} \cos 3 \phi+O\left(A^{5}\right) \\
& \frac{\left(F_{0}+F_{90}+F_{180}+F_{270}\right)}{4}=A^{2} f_{20}+A^{4} f_{44} \cos 4 \phi+O\left(A^{6}\right) .
\end{aligned}
$$

provided by Fitzgerald et al. [39]. Here the superscript $H$ shows the harmonic conjugate of the signal, obtained by Hilbert transformation. Equations (11) to (13) separate the first three harmonics of the signal while equation (14) includes the second-order difference long-wave force signal and the fourth-order summation harmonic force signal which are easily distinguished in the frequency domain.

The time series of different harmonics were separated and plotted on a sloped bed versus the equivalent flat bed for all sea states and each target force. The results for sea state 4 with the largest target force are presented in figure 19. In this figure it is visible that the differences between the time series on a sloped bed versus the flat bed are negligible in the main wave group envelope. However, deviations are observed between the time series of the second- and thirdorder free surface elevation and inline force for the two bed configurations. The differences in the second packet of 
the waves in higher orders is related to the free spurious waves caused by the linear boundary condition. In the cases on a sloped bed this linear boundary condition is more accurate because of the larger depth at the location of the wave makers. Hence, smaller spurious waves are created. The same consistency between the results from the flat bed and the sloped bed around the inline force peak time are also observed in other cases in sea states 3 to 8 . In the time series of harmonics of free surface elevation and inline force, differences between the cases on flat and sloped bed are visible further away from the peak time of the total inline force in the sea states 6, 7 and 8 which have larger Ursell numbers. In all of the cases the waves on a flat bed contained larger packets of spurious waves than the counterpart waves on the sloped bed. This observation is in consistency with the comparisons of the PSDs of these cases, where the frequency content of the cases on a flat bed was larger than the cases on a sloped bed around the second harmonic frequency range.
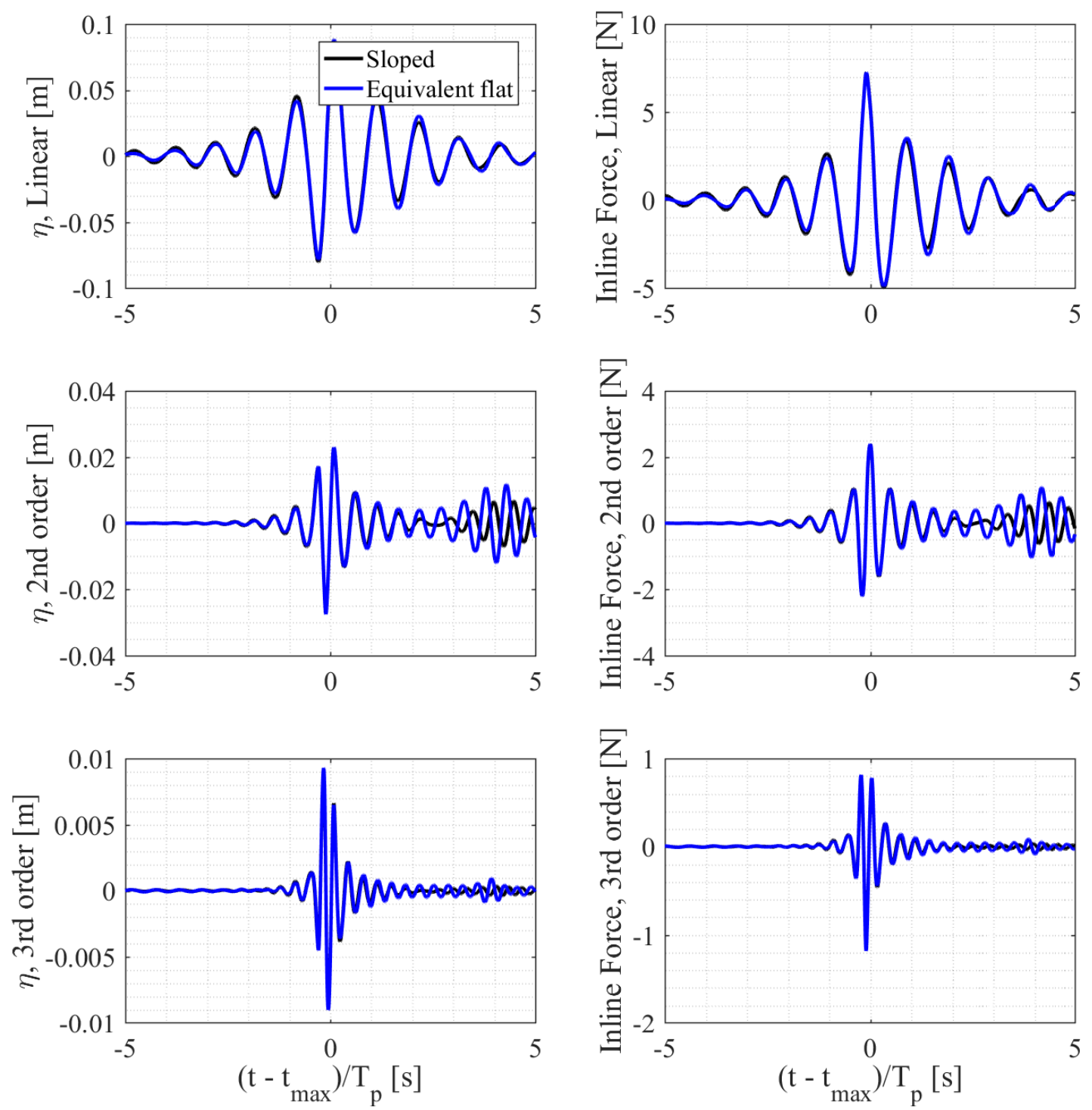

Figure 19: Time series of free surface elevation and inline force of different harmonics for sea state 4 with target inline force $1.2 \rho g h R^{2}$ 
In figure 20 the same time series as in the figure 19 are shown but zoomed in around the time for the main force peak. The plots show that the different harmonics of the free surface elevation reach their peak at the same time relative to each other. The peak time, however, changes relative to the time axis zero as a function of target forces and sea states. Further, the different harmonics of the inline force time series have time shifts relative to each other which are fixed among all target forces and sea states. The peak times, similarly to the free surface elevation, do shift in relation to the total inline force time series peak time for different target forces. There is a $90^{\circ}$ phase shift between the linear harmonic of $\eta$ and $F$ which is expected from the linear theory. This phase shift is smaller for higher harmonics. These unique observations, which are irrespective of the bed slope, can help to develop an analytical force model similar to the model developed by Sarkar et al. [40].
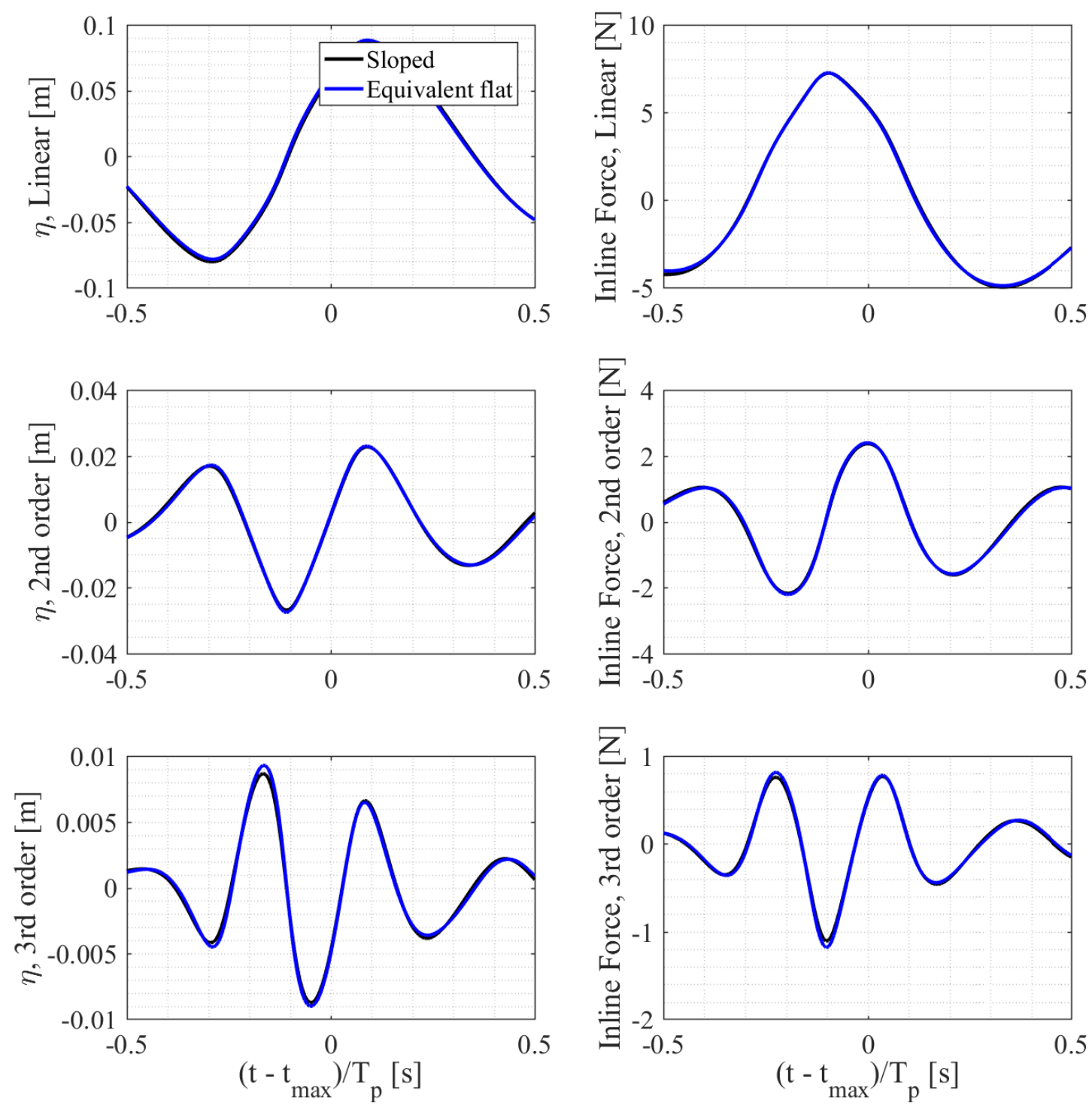

Figure 20: Time series of free surface elevation and inline force of different harmonics for sea state 4 with target inline force $1.2 \rho g h R^{2}$ with limited $\mathrm{x}$ axis. 
In order to investigate the change of the magnitude of different harmonics in different sea states and with different target forces, the relative contribution of the inline force value from the different harmonics at the peak time of the total inline force time series to the target inline force was calculated. This was quantified as

$$
\text { Relative contribution of n'th harmonic }=\frac{F_{n}\left(t_{\text {max }}\right)}{F_{\text {total }}\left(t_{\text {max }}\right)}
$$

where $n$ is harmonic number and $t_{\max }$ is the time when the total force peaks.

Table 2 to 4 show these ratios for three different sea states, 4, 6 and 8, on flat and sloped bed, for different target force values. In table 2 these results are shown for sea state 4 . It can be seen that the relative linear contribution decreases as the target force in that sea state increases. As the relative linear contribution decreases the relative contribution of the 2 nd and 3rd order superharmonic increase. The 2nd order subharmonic contribution has the smallest effect while the 2 nd order superharmonic has the largest effect on the final inline force after the linear contribution.

Further, the results show that the differences between the linear and nonlinear contributions on a sloped and equivalent flat bed are negligible. This observation is true despite the fact that the probability of exceedance is different for each target value in this sea state, see figure A.35.

Table 2: Ratio of the relative contribution of different harmonics on the inline force at the target time in sea state 4 on sloped and equivalent flat bed.

\begin{tabular}{llllllll}
\hline $\begin{array}{c}\text { Target force } \\
{\left[\rho g h R^{2}\right]}\end{array}$ & Bed slope & Linear & 2nd order super- & 2nd order sub- & 3rd order & 4th order & Total \\
\hline \multirow{2}{*}{0.9} & sloped & $8.0 \cdot 10^{-1}$ & $2.2 \cdot 10^{-1}$ & $1.0 \cdot 10^{-2}$ & $-1.0 \cdot 10^{-2}$ & $-2.0 \cdot 10^{-2}$ & 1.0 \\
& flat & $8.2 \cdot 10^{-1}$ & $2.2 \cdot 10^{-1}$ & 0.0 & $-1.0 \cdot 10^{-2}$ & $-2.0 \cdot 10^{-2}$ & 1.0 \\
\hline \multirow{2}{*}{1.0} & sloped & $7.7 \cdot 10^{-1}$ & $2.4 \cdot 10^{-1}$ & $1.0 \cdot 10^{-2}$ & $1.0 \cdot 10^{-2}$ & $-2.0 \cdot 10^{-2}$ & 1.0 \\
& flat & $7.6 \cdot 10^{-1}$ & $2.5 \cdot 10^{-1}$ & $1.0 \cdot 10^{-2}$ & 0.0 & $-2.0 \cdot 10^{-2}$ & 1.0 \\
\hline \multirow{2}{*}{1.1} & sloped & $7.2 \cdot 10^{-1}$ & $2.7 \cdot 10^{-1}$ & $1.0 \cdot 10^{-2}$ & $3.0 \cdot 10^{-2}$ & $-2.0 \cdot 10^{-2}$ & 1.0 \\
& flat & $7.1 \cdot 10^{-1}$ & $2.7 \cdot 10^{-1}$ & $1.0 \cdot 10^{-2}$ & $3.0 \cdot 10^{-2}$ & $-2.0 \cdot 10^{-2}$ & 1.0 \\
\hline \multirow{2}{*}{1.2} & sloped & $6.6 \cdot 10^{-1}$ & $2.8 \cdot 10^{-1}$ & $2.0 \cdot 10^{-2}$ & $5.0 \cdot 10^{-2}$ & 0.0 & 1.0 \\
& flat & $6.5 \cdot 10^{-1}$ & $2.8 \cdot 10^{-1}$ & $2.0 \cdot 10^{-2}$ & $5.0 \cdot 10^{-2}$ & 0.0 & 1.0 \\
\hline
\end{tabular}

In table 3 the relative contribution of the different harmonics is presented for sea state 6 on a sloped and equivalent flat bed. The contribution of the different harmonics follow the same pattern as sea state 4 . The relative linear contribution decreases by increased target force while the relative contribution of the higher harmonics increase. The different harmonic contributions between the two bed slopes is very similar. The largest difference between the contributions of different harmonics is observed between the linear contribution for the largest target force in which the linear contribution is $6 \%$ smaller on the flat bed. The breaking filter was activated close to the structure and at the peak time of the inline force which might explain the smaller contribution from the linear harmonic in this case.

Table 3: Ratio of the relative effect of different harmonics on the inline force at the target time. sea state 6 sloped and equivalent flat bed.

\begin{tabular}{llllllll}
\hline $\begin{array}{c}\text { Target force } \\
{\left[\rho g h R^{2}\right]}\end{array}$ & Bed slope & Linear & 2nd order super- & 2nd order sub- & 3rd order & 4th order & Total \\
\hline \multirow{2}{*}{1.1} & slope & $6.4 \cdot 10^{-1}$ & $3.2 \cdot 10^{-1}$ & 0.0 & $6.0 \cdot 10^{-2}$ & $-1.0 \cdot 10^{-2}$ & 1.0 \\
& flat & $6.2 \cdot 10^{-1}$ & $3.2 \cdot 10^{-1}$ & 0.0 & $7.0 \cdot 10^{-2}$ & $-1.0 \cdot 10^{-2}$ & 1.0 \\
\hline 1.3 & slope & $5.5 \cdot 10^{-1}$ & $3.3 \cdot 10^{-1}$ & 0.0 & $1.0 \cdot 10^{-1}$ & $1.0 \cdot 10^{-2}$ & $9.9 \cdot 10^{-1}$ \\
& flat & $5.4 \cdot 10^{-1}$ & $3.3 \cdot 10^{-1}$ & 0.0 & $1.1 \cdot 10^{-1}$ & $2.0 \cdot 10^{-2}$ & 1.0 \\
\hline \multirow{2}{*}{1.5} & slope & $4.8 \cdot 10^{-1}$ & $3.3 \cdot 10^{-1}$ & $1.0 \cdot 10^{-2}$ & $1.3 \cdot 10^{-1}$ & $5.0 \cdot 10^{-2}$ & 1.0 \\
& flat & $4.9 \cdot 10^{-1}$ & $3.3 \cdot 10^{-1}$ & $-1.0 \cdot 10^{-2}$ & $1.3 \cdot 10^{-1}$ & $5.0 \cdot 10^{-2}$ & $9.9 \cdot 10^{-1}$ \\
\hline \multirow{2}{*}{1.7} & slope & $4.4 \cdot 10^{-1}$ & $3.2 \cdot 10^{-1}$ & $1.0 \cdot 10^{-2}$ & $1.5 \cdot 10^{-1}$ & $8.0 \cdot 10^{-2}$ & 1.0 \\
& flat & $4.1 \cdot 10^{-1}$ & $3.2 \cdot 10^{-1}$ & 0.0 & $1.6 \cdot 10^{-1}$ & $1.1 \cdot 10^{-1}$ & 1.0 \\
\hline
\end{tabular}


In table 4 the relative contribution of different harmonics is presented for sea state 8 on a sloped and equivalent flat bed. Similarly to the previous cases the contribution of the first harmonic decreases by the increase of the target force while the contribution of the higher harmonics increases. However, the contribution of the second harmonic seems to be constant in all the target forces on flat or sloped bed.

Table 4: Ratio of the relative effect of different harmonics on the inline force at the target time. sea state 8 sloped and equivalent flat bed

\begin{tabular}{llllllll}
\hline $\begin{array}{c}\text { Target force } \\
{\left[\rho g h R^{2}\right]}\end{array}$ & Bed slope & Linear & 2nd order super- & 2nd order sub- & 3rd order & 4th order & Total \\
\hline \multirow{2}{*}{1.3} & slope & $4.8 \cdot 10^{-1}$ & $3.6 \cdot 10^{-1}$ & $-2.0 \cdot 10^{-2}$ & $1.5 \cdot 10^{-1}$ & $3.0 \cdot 10^{-2}$ & 1.0 \\
& flat & $4.9 \cdot 10^{-1}$ & $3.6 \cdot 10^{-1}$ & $-3.0 \cdot 10^{-2}$ & $1.5 \cdot 10^{-1}$ & $3.0 \cdot 10^{-2}$ & 1.0 \\
1.4 & slope & $4.5 \cdot 10^{-1}$ & $3.5 \cdot 10^{-1}$ & $-2.0 \cdot 10^{-2}$ & $1.7 \cdot 10^{-1}$ & $5.0 \cdot 10^{-2}$ & 1.0 \\
& flat & $4.7 \cdot 10^{-1}$ & $3.6 \cdot 10^{-1}$ & $-3.0 \cdot 10^{-2}$ & $1.6 \cdot 10^{-1}$ & $4.0 \cdot 10^{-2}$ & 1.0 \\
1.5 & slope & $4.3 \cdot 10^{-1}$ & $3.5 \cdot 10^{-1}$ & $-2.0 \cdot 10^{-2}$ & $1.8 \cdot 10^{-1}$ & $6.0 \cdot 10^{-2}$ & 1.0 \\
& flat & $4.6 \cdot 10^{-1}$ & $3.6 \cdot 10^{-1}$ & $-4.0 \cdot 10^{-2}$ & $1.7 \cdot 10^{-1}$ & $5.0 \cdot 10^{-2}$ & 1.0 \\
1.6 & slope & $4.1 \cdot 10^{-1}$ & $3.4 \cdot 10^{-1}$ & $-2.0 \cdot 10^{-2}$ & $1.9 \cdot 10^{-1}$ & $8.0 \cdot 10^{-2}$ & 1.0 \\
& flat & $4.4 \cdot 10^{-1}$ & $3.5 \cdot 10^{-1}$ & $-4.0 \cdot 10^{-2}$ & $1.8 \cdot 10^{-1}$ & $6.0 \cdot 10^{-2}$ & $9.9 \cdot 10^{-1}$ \\
\hline
\end{tabular}

\section{Discussion and future work}

We have investigated the effect of sea bed slope on the force statistics and the shapes of the force time series numerically using the nonlinear wave model, OceanWave3D, and FORM analysis. The wave and FORM model were initially validated against two sets of measured data. The validations were also compared with previously published results in which first- and second-order wave theories were used instead of the fully nonlinear flow solver and it was shown that a significant improvement is obtained using the new method.

It was shown that the First Order Reliability Method in combination with the OceanWave3D potential flow solver can be used to estimate the most probable wave that creates a predefined target inline force. The comparisons with the ensemble averaged measurements showed the validity of this method. The exceedance probability calculated from this method was compared to random realizations of the same sea states and good consistency between the numerical probabilistic approach, the random deterministic approach and the measurements was observed.

The validated wave model and FORM setup was next used to compare the extreme force episodes of the two bed slopes. The time series and power spectral density of these force episodes were compared between the two bed configurations. It was shown that the frequency contents in the linear range are very similar for low Ursell number while the differences are larger when the Ursell number is larger. The frequency contents in the range of the secondharmonic peak was always larger on the equivalent flat beds and can be linked to the larger occurance of spurious second-order waves at flat bed due to the linear wave generation technique. The exceedance probability of the cases on flat and sloped beds were also compared for all the target maximum inline forces in all the 6 sea states. It was observed that in the same sea state, on a sloped bed the probability of exceedance for a given maximum inline force is larger. The ratio of the exceedance probability between the sloped and flat bed was calculated and it was observed that in all the sea states with lower Ursell number the ratio increases with force level while in the sea states with larger Ursell number, in our case $\mathrm{Ur} \geq 20$, the trend is not as uniform because of larger nonlinearities but it can be larger than 2.

From the harmonic separation method it was observed that the relative contribution of the higher harmonics in each sea state increases as the target force is increased while the relative linear contribution decreases in all cases. The higher harmonic spurious waves were found to be more significant for the cases on flat bed. Moreover, it was shown that the relative contributions of the higher harmonics to the maximum inline force of the waves are not influenced significantly by the bed slope. The phases of the different harmonics of the inline force and free surface elevation histories are locked to each other with constant phase shifts irrespective of the bed slope. The free surface elevation time series of different harmonics all peaked simultaneously in all cases. 
It should be noted that the suggested method in this paper is an alternative to the use of Monte Carlo simulations. Even though the suggested method is not computationally cheap it is cheaper than using Monte Carlo method to investigate the effect of the bed slope on the extreme waves. The current method can be used in the design process to provide the designer with extreme nonlinear waves that create a certain inline force or bending moment with a given exceedance probability. The method could also be used for response of the structures. Based on the current study the slope effect on exceedance probability is significant and calls for care in the analysis of lab test results. To extend the research a wider range of sea states, with different water depths and different bed slopes can be investigated. In addition, the method can be used for bed slopes with larger shoaling coefficient where the wave spectrum change from the paddle position to the location of the structure is also of interest. The fixed phases between the different harmonics in the inline force time series can be calculated and used to formulate new analytical wave force models based on the model suggested by Sarkar et al. [40].

\section{Acknowledgement}

This work was funded by the Innovation Fund Denmark and other partners as part of DeRisk project with grant number 4106-00038B. This support is gratefully acknowledged by the authors. The authors are also grateful to professor Paul Taylor, the university of Western Australia, associate professor Thomas Adcock, the university of Oxford, professor emeritus Jørgen Juncher Jensen, Denmark Technical University and Ju Hyuck Choi, Denmark Technical University for helpful discussions.

\section{References}

[1] Peter S Tromans, A R Anatruk, and Paul Hagemeijer. A New Model for the Kinematics of Large Ocean Waves Application as a Design Wave. Proceedings of the First International Offshore and Polar Engineering Conference, 8(August):64-71,1991.

[2] J.D. Fenton. The numerical solution of steady water wave problems. Computers $\mathcal{E}$ Geosciences, 14(3):357-368, 1988.

[3] IEC 61400-3. 2009.

[4] R. C. Nelson. The Effect of Bed Slope on Wave Characteristics. pages 555-572, 1982.

[5] Steve Elgar and R. T. Guza. Shoaling gravity waves: comparisons between field observations, linear theory, and a nonlinear model, 1985.

[6] M H Freilich and R. T. Guza. Nonlinear Effects on Shoaling Surface Gravity Waves. 41:1-41, 1984.

[7] M H Freilich, R T Guza, and S L Elgar. Observations of Nonlinear Effects in Directional Spectra of Shoaling Gravity Waves. J. Geophysical Res., 95(C6):9645-9656, 1990.

[8] Okey Nwogu. Alternative Form of Boussinesq Equations for Nearshore Wave Propagation. Journal of Waterway, Port, Coastal, and Ocean Engineering, 119(6):618-638, 1993.

[9] S. Schløer, H. Bredmose, and H. B. Bingham. Irregular Wave Forces on Monopile Foundations: Effect of Full Nonlinearity and Bed Slope. International Conference on Ocean, Offshore and Arctic Engineering, 2011.

[10] V. Katsardi, L. de Lutio, and C. Swan. An experimental study of large waves in intermediate and shallow water depths. Part I: Wave height and crest height statistics. Coastal Engineering, 73:43-57, 2013.

[11] Alberto Alberello, Amin Chabchoub, Jason P. Monty, Filippo Nelli, Jung Hoon Lee, John Elsnab, and Alessandro Toffoli. An experimental comparison of velocities underneath focussed breaking waves. Ocean Engineering, 155:201-210, may 2018.

[12] Peter Friis Hansen and Lars P Nielsen. On the new wave model for the kinematics of large ocean waves. Proceedings of the International Conference on Offshore Mechanics and Arctic Engineering - Omae, 1(Part A), 1995.

[13] Jørgen Juncher Jensen. Second-order wave kinematics conditional on a given wave crest. Applied Ocean Research, 18(2-3):119-128, 1996.

[14] R. Torhaug. Extreme Response Analysis of Non-linear Ocean Structures: Identification of Minimal Stochastic Wave Input for Time Domain Simulation. PhD thesis, Stanford University, 1996.

[15] LJM Adegeest, A Braathen, and RM Loseth. Use of non-linear sea-loads simulations in design of ships. In Proceedings PRADS conference, volume 11, page 1089. Elsevier, 1998

[16] L.W. PASTOOR. On the assessment of nonlinear ship motions and loads. PhD thesis, Technische Universiteit Delft (The Netherlands), 2002.

[17] Signe Schløer, Henrik Bredmose, and Amin Ghadirian. Analysis of experimental data: The average shape of extreme wave forces on monopile foundations. In DeepWind, volume 137, pages 223-237. Elsevier, oct 2017.

[18] Amin Ghadirian, Henrik Bredmose, and Signe Schløer. Prediction of the shape of inline wave force and free surface elevation using First Order Reliability Method ( FORM ). Energy Procedia, 00(January):18-20, oct 2017.

[19] H. Bredmose, M. Dixen, A. Ghadirian, T.J. Larsen, S. Schløer, S.J. Andersen, S. Wang, H.B. Bingham, O. Lindberg, E.D. Christensen, M.H. Vested, S. Carstensen, A.P. Engsig-Karup, O.S. Petersen, H.F. Hansen, J.S. Mariegaard, P.H. Taylor, T.A.A. Adcock, C. Obhrai, O.T. Gudmestad, N.J. Tarp-Johansen, C.P. Meyer, J.R. Krokstad, L. Suja-Thauvin, and T.D. Hanson. DeRisk - Accurate prediction of ULS wave loads. Outlook and first results. In Energy Procedia, volume 94, pages 379-387. Elsevier, sep 2016.

[20] Henrik Bredmose, Jesper Mariegaard, Bo Terp Paulsen, Bjarne Jensen, Signe Schløer, Torben Larsen, Taeseong Kim, and Anders Melchior Hansen. The Wave Loads project. 2013.

[21] Robert Dean. Water wave mechanics for engineers and scientists, volume 53. 2013

[22] D T U Vindenergi. DeRisk - WP2 Proposal for DeRisk 3D Physical Model Tests DTU Vindenergi. (October), 2015. 
[23] H. Bredmose. Dynamic excitation of monopiles by steep and breaking waves. Experimental and numerical study. In OMAE2010: Proceedings of the ASME 29th 2010 International Conference on Ocean, Offshore and Arctic Engineering, number 2011, 2013.

[24] F. Ursell. The long-wave paradox in the theory of gravity waves. Mathematical Proceedings of the Cambridge Philosophical Society, 49(04):685, oct 1953

[25] Yoshimi Goda and Yasumasa Suzuki. Estimation of Incident and Reflected Waves in Random Wave Experiments. In Proc. of 15th Int. Conference on Coastal Engineering, ASCE, pages 828-845, 1976.

[26] H. Bredmose, A. Hunt-Raby, R. Jayaratne, and G. N. Bullock. The ideal flip-through impact: Experimental and numerical investigation. Journal of Engineering Mathematics, 67(1):115-136, 2010.

[27] Yoshimi Goda. Reanalysis of Regular and Random Breaking Wave Statistics. Coastal Engineering Journal, 52(01):71-106, 2010.

[28] J R Grice, P H Taylor, and R Eatock Taylor. Second-order statistics and designer' waves for violent free-surface motion around multi-column structures Subject Areas :. 2014.

[29] A. P. Engsig-Karup, H. B. Bingham, and O. Lindberg. An efficient flexible-order model for 3D nonlinear water waves. Journal of Computational Physics, 228(6):2100-2118, 2009.

[30] Bo Terp Paulsen, Henrik Bredmose, and Harry B. Bingham. An efficient domain decomposition strategy for wave loads on surface piercing circular cylinders. Coastal Engineering, 86:57-76, 2014.

[31] Bo T. Paulsen, H. Bredmose, H. B. Bingham, and N. G. Jacobsen. Forcing of a bottom-mounted circular cylinder by steep regular water waves at finite depth. Journal of Fluid Mechanics, 755:1-34, 2014.

[32] R. C. T. Rainey. Slender-body expressions for the wave load on offshore structures. Proceedings of the Royal Society of London, 450(1939):391-416, 1995.

[33] Sylvain Lacaze and Samy Missoum. A generalized "max-min" sample for surrogate update. Structural and Multidisciplinary Optimization, 49(4):683-687, 2014.

[34] H.C. Longuet-Higgins. On the statistical distribution of the height of sea waves. The Journal of Japan Institute of Navigation, 11:245-266, 1952.

[35] R. S. Gibson, C. Swan, and P. S. Tromans. Fully Nonlinear Statistics of Wave Crest Elevation Calculated Using a Spectral Response Surface Method: Applications to Unidirectional Sea States. Journal of Physical Oceanography, 37(2000):3-15, 2007.

[36] Jørgen Juncher Jensen. Efficient estimation of extreme non-linear roll motions using the first-order reliability method (FORM). Journal of Marine Science and Technology, 12(4):191-202, 2007.

[37] John Grue and Morten Huseby. Higher harmonic wave forces and ringing of vertical cylinders. Applied Ocean Research, 24(4):203-214, 2002.

[38] Bradley Efron. An introduction to the Bootstrap. 1993.

[39] C. Fitzgerald, Paul H. Taylor, R. Eatock Taylor, J. Grice, and Jun Zang. Phase manipulation and the harmonic components of ringing forces on a surface piercing column. Proceedings of the Royal Society of London A: Mathematical, Physical and Engineering Sciences, 470(2168):20130847, 2014

[40] Dripta Sarkar, Paul H Taylor, Thomas A A Adcock, and Henrik Bredmose. The extraction of nonlinear harmonics of extreme wave load on a surface piercing column in random waves. Journal of Fluid Mechanics, (Submited for publication), 2017. 


\section{Appendix A. Additional results}

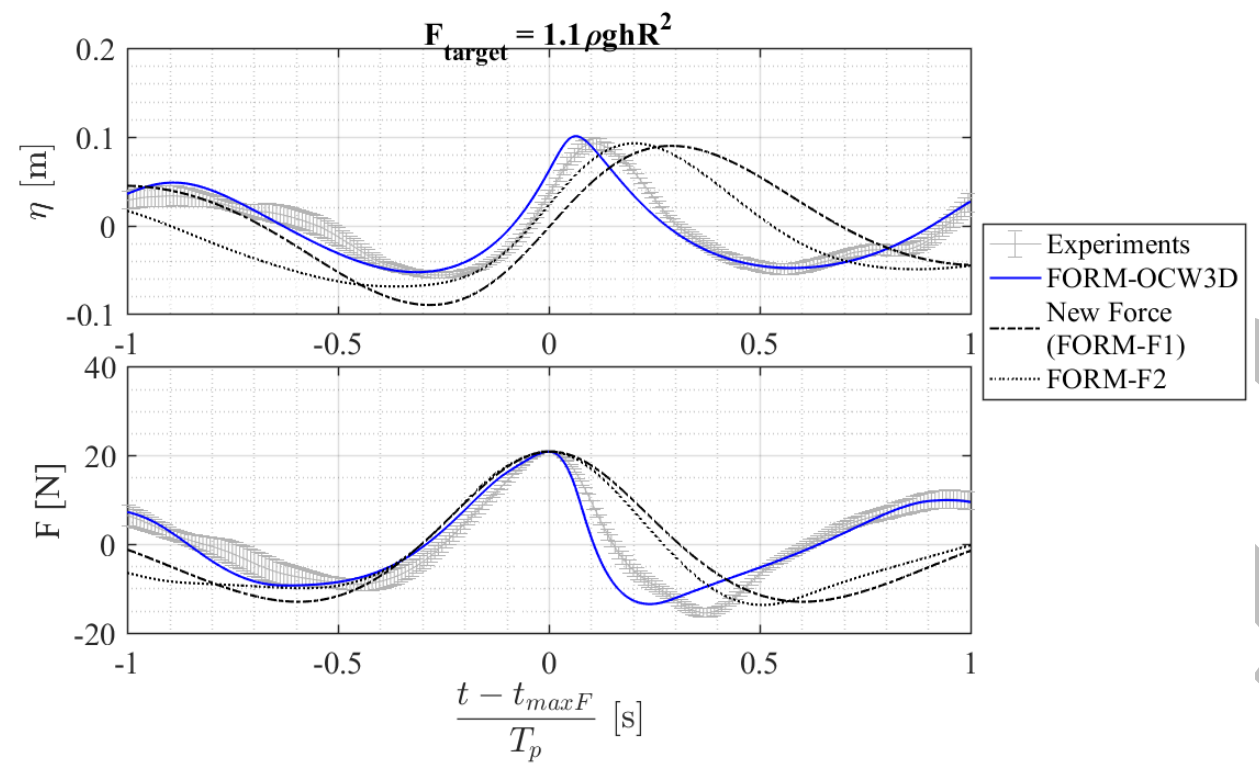

Figure A.21: Free surface elevation and inline force time series of the sea state $2, F_{\text {target }}=1.1 \cdot \rho g h R^{2}$, on a flat bed.

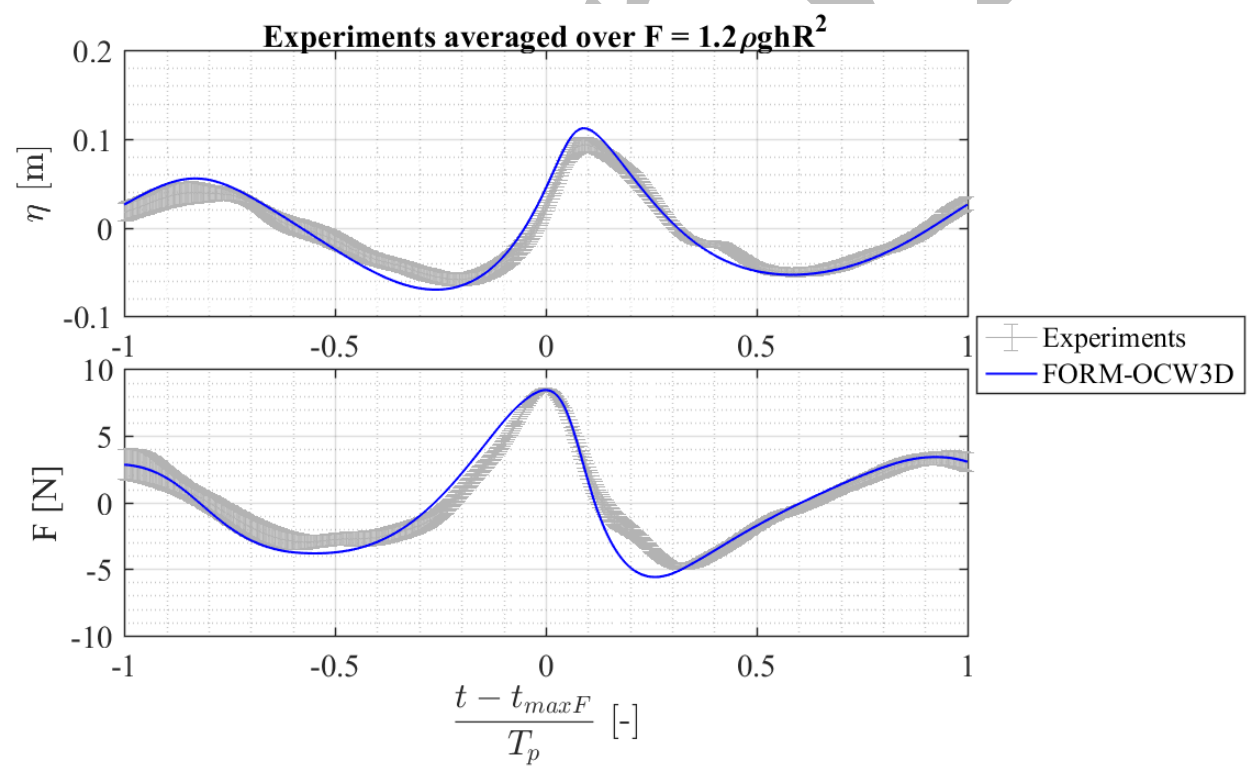

Figure A.22: Free surface elevation and inline force time series of the sea state $4, F_{\text {target }}=1.2 \cdot \rho g h R^{2}$, on a sloped bed. 


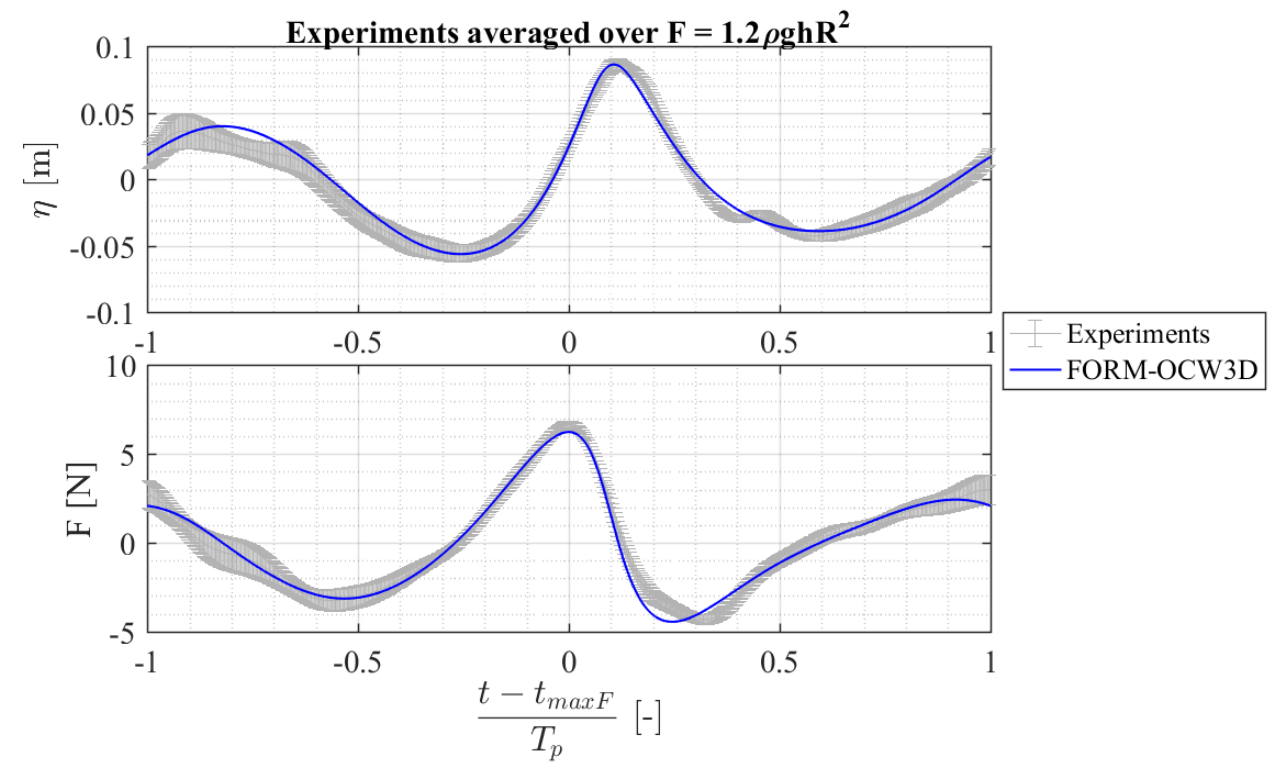

Figure A.23: Free surface elevation and inline force time series of the sea state $5, F_{\text {target }}=1.2 \cdot \rho g h R^{2}$, on a sloped bed.

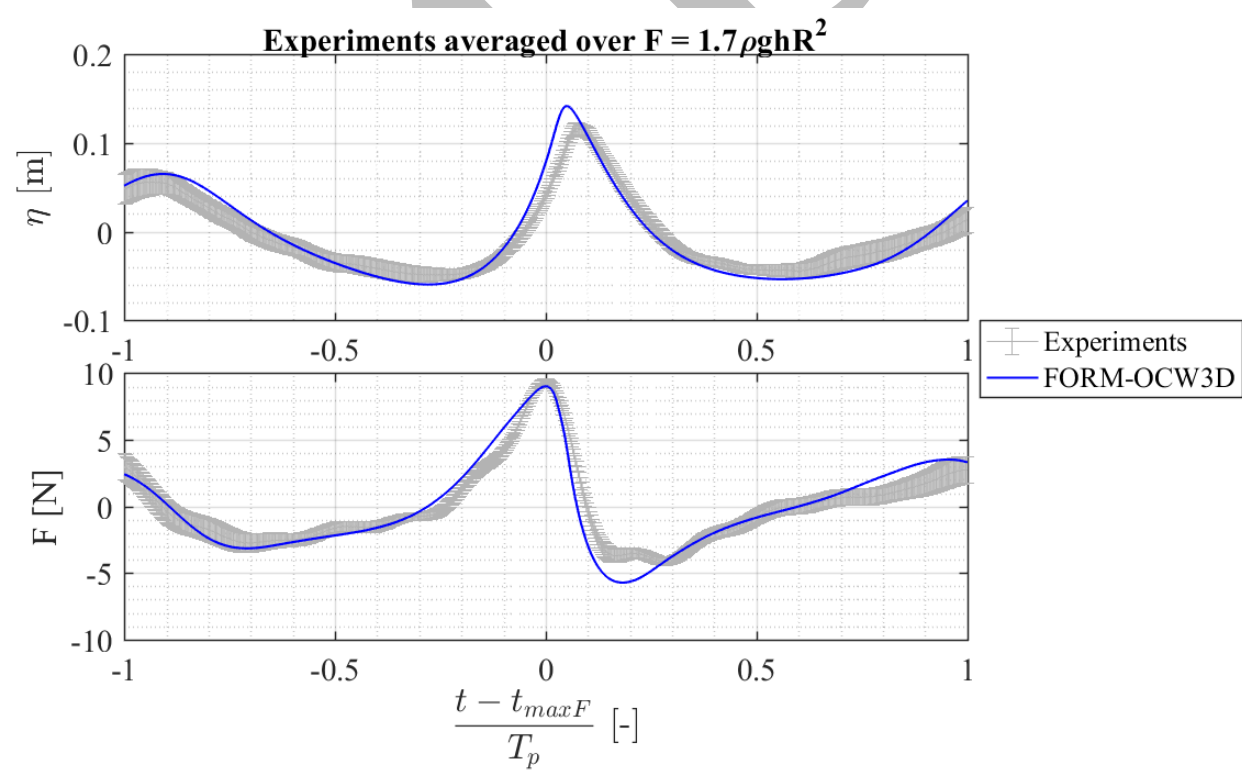

Figure A.24: Free surface elevation and inline force time series of the sea state $6, F_{\text {target }}=1.7 \cdot \rho g h R^{2}$, on a sloped bed. 


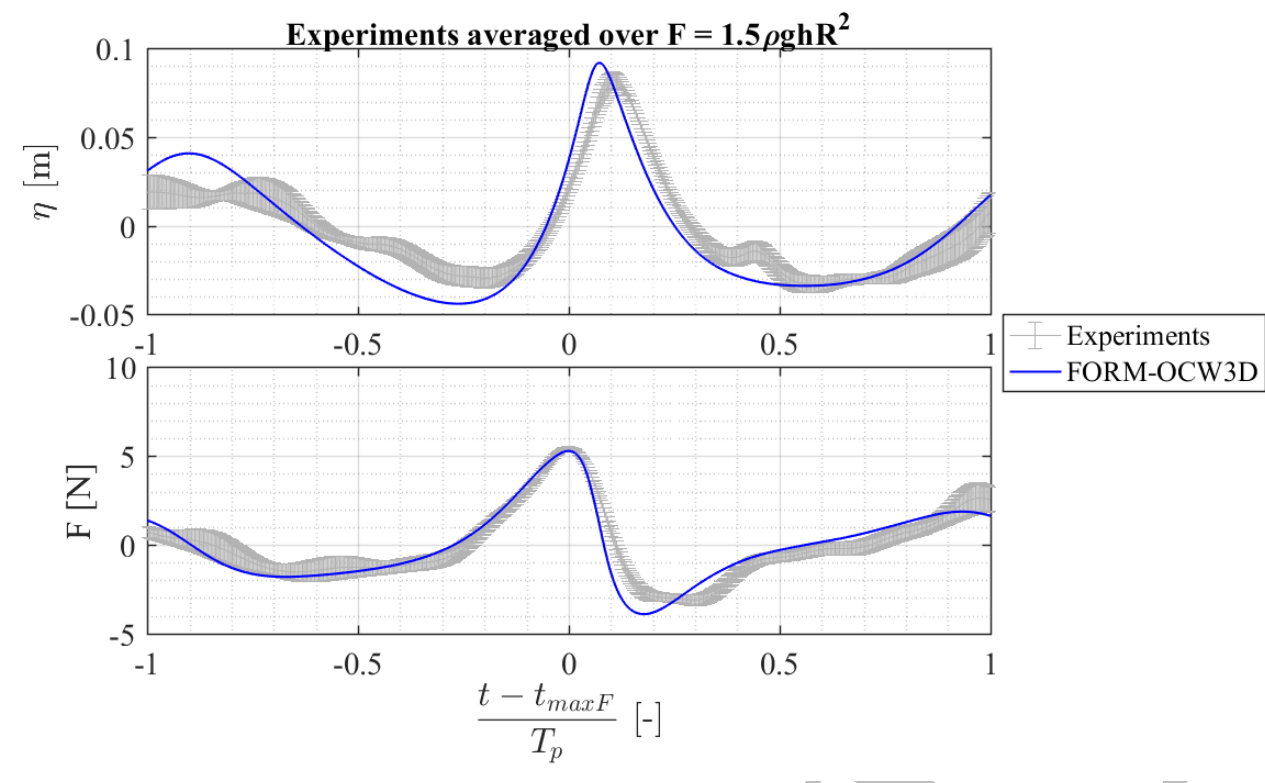

Figure A.25: Free surface elevation and inline force time series of the sea state $7, F_{\text {target }}=1.5 \cdot \rho g h R^{2}$, on a sloped bed.

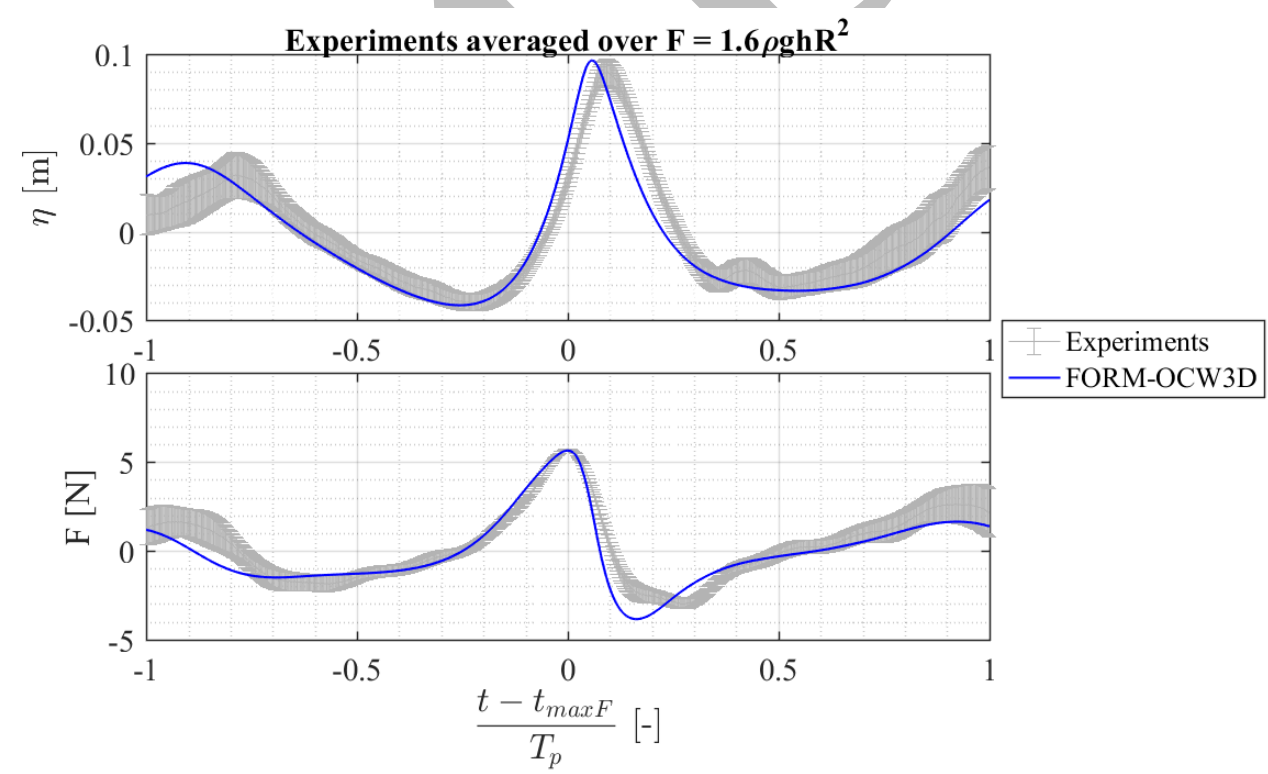

Figure A.26: Free surface elevation and inline force time series of the sea state $8, F_{\text {target }}=1.6 \cdot \rho g h R^{2}$, on a sloped bed. 

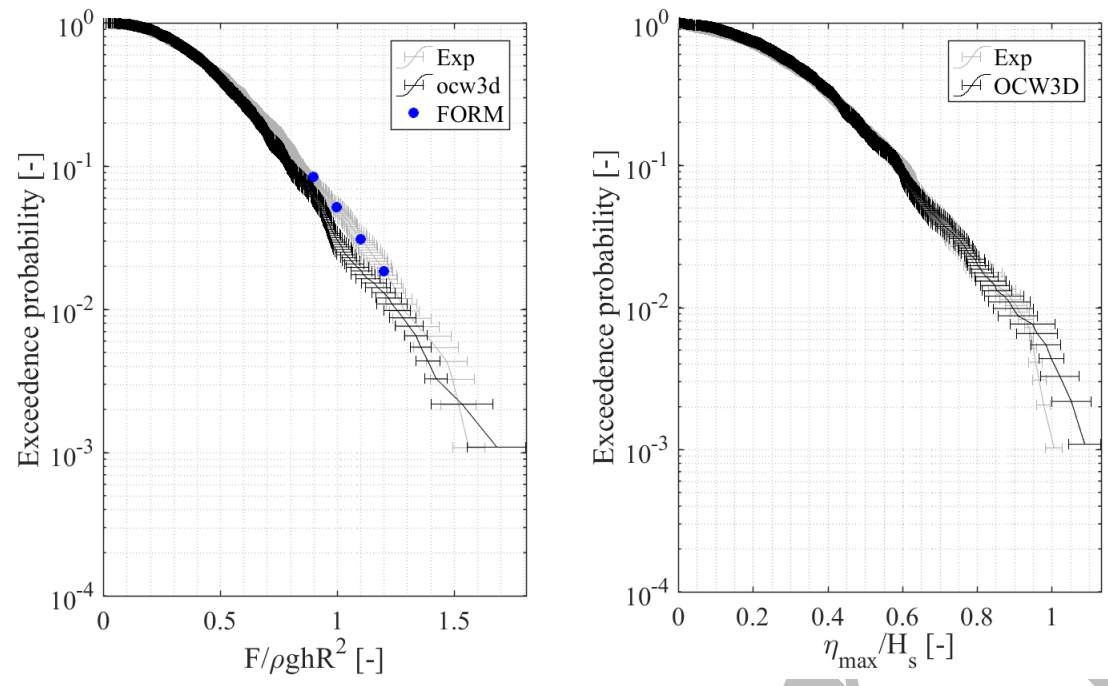

Figure A.27: Exceedance probabilities relative to maximum inline force from random realization tests, numerical simulations in OceanWave3D and numerical results from FORM probem for sea states of 4 on sloped bed.
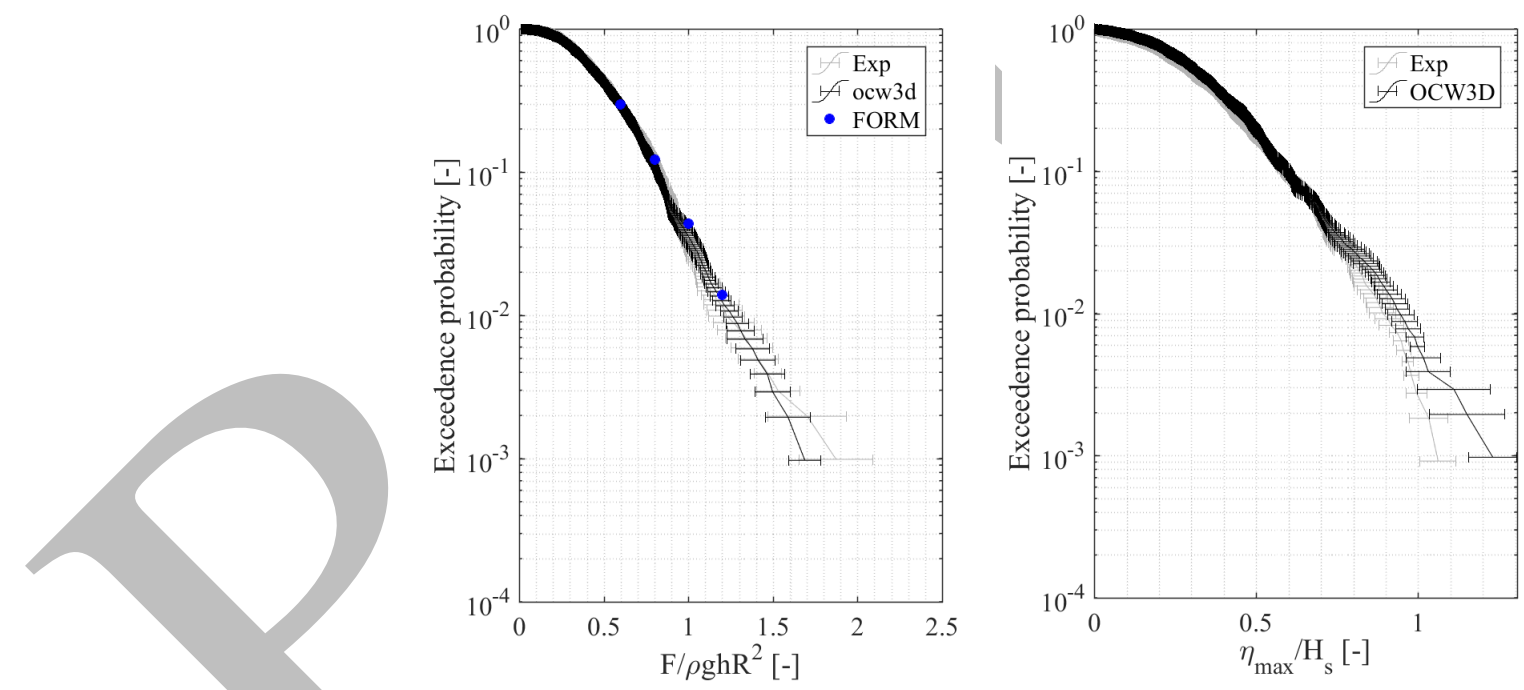

Figure A.28: Exceedance probabilities relative to maximum inline force from random realization tests, numerical simulations in OceanWave3D and numerical results from FORM probem for sea states of 5 on sloped bed. 

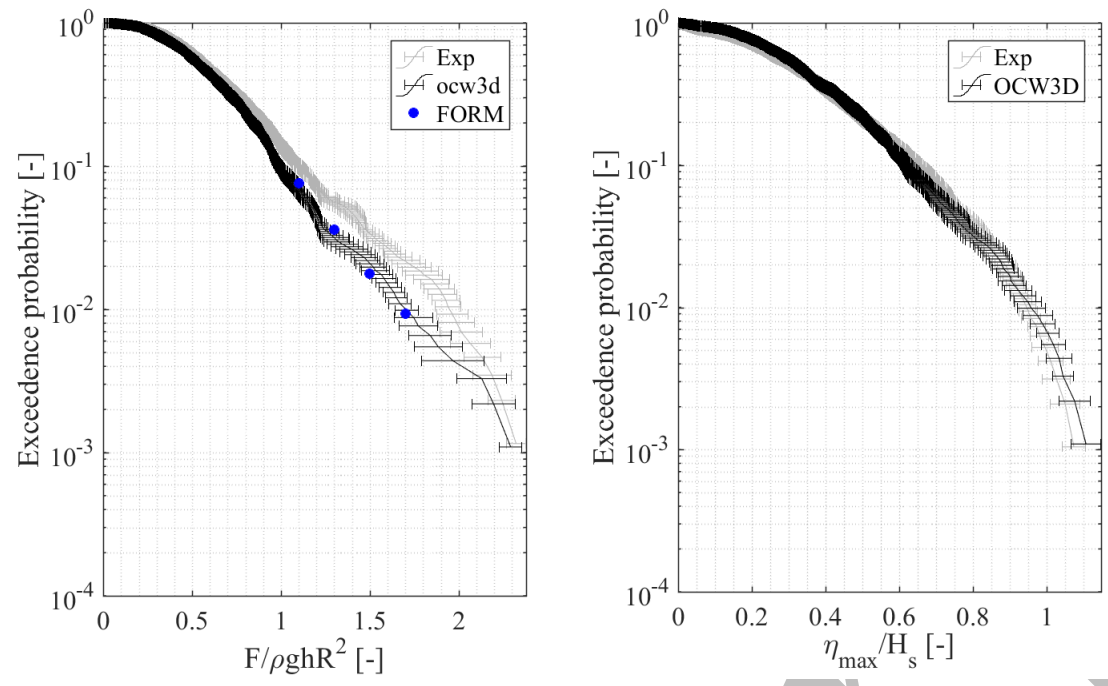

Figure A.29: Exceedance probabilities relative to maximum inline force from random realization tests, numerical simulations in OceanWave3D and numerical results from FORM probem for sea states of 6 on sloped bed.
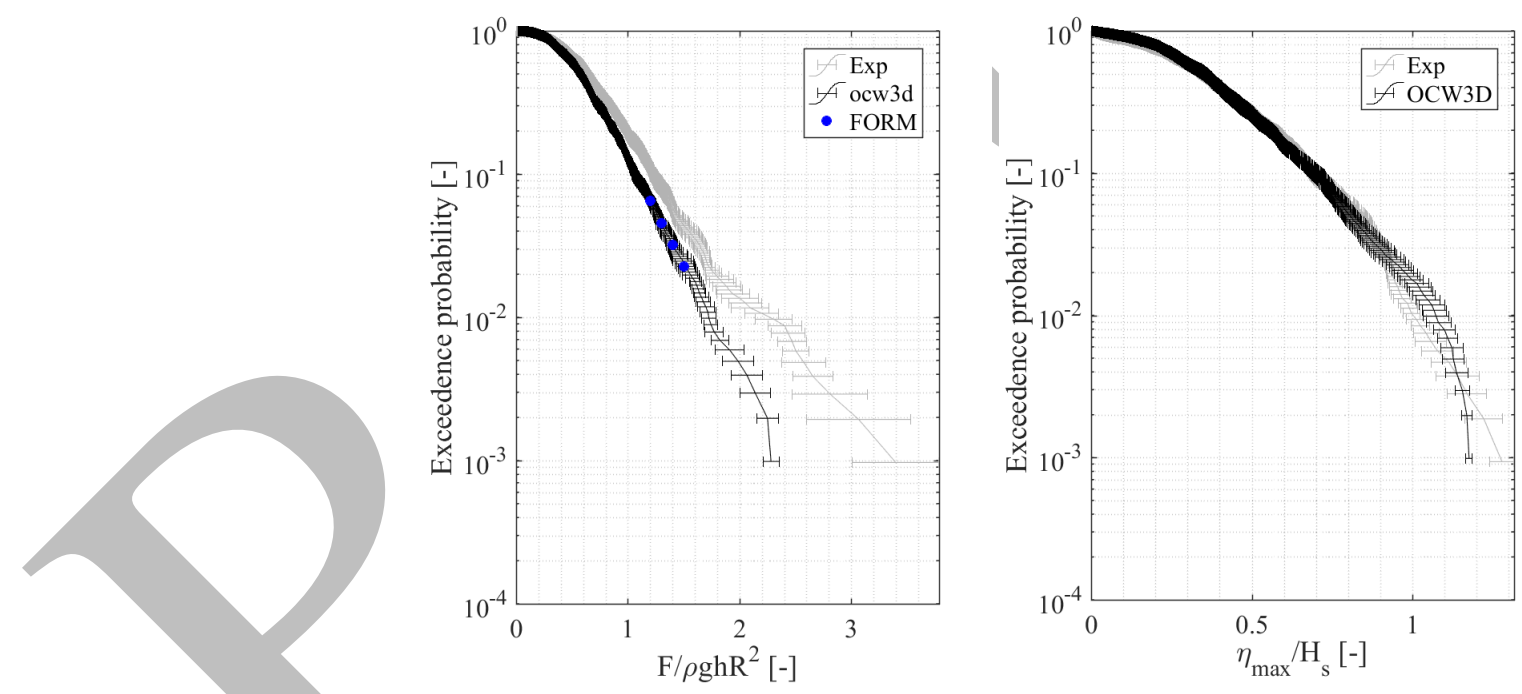

Figure A.30: Exceedance probabilities relative to maximum inline force from random realization tests, numerical simulations in OceanWave3D and numerical results from FORM probem for sea states of 7 on sloped bed. 

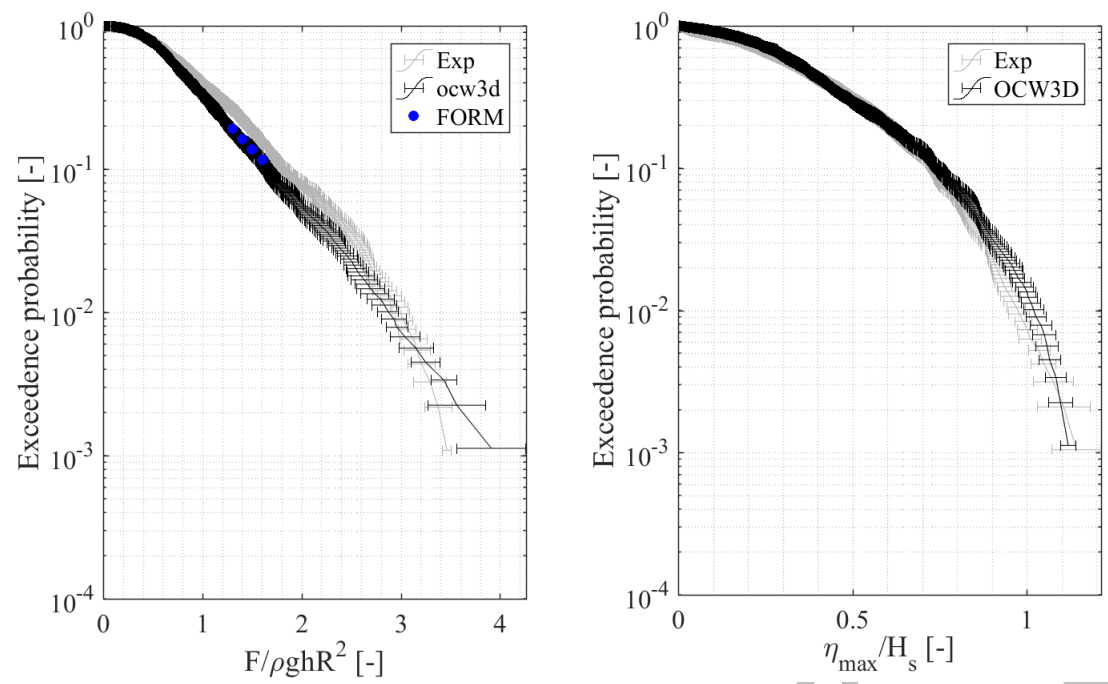

Figure A.31: Exceedance probabilities relative to maximum inline force from random realization tests, numerical simulations in OceanWave3D and numerical results from FORM probem for sea states of 8 on sloped bed.
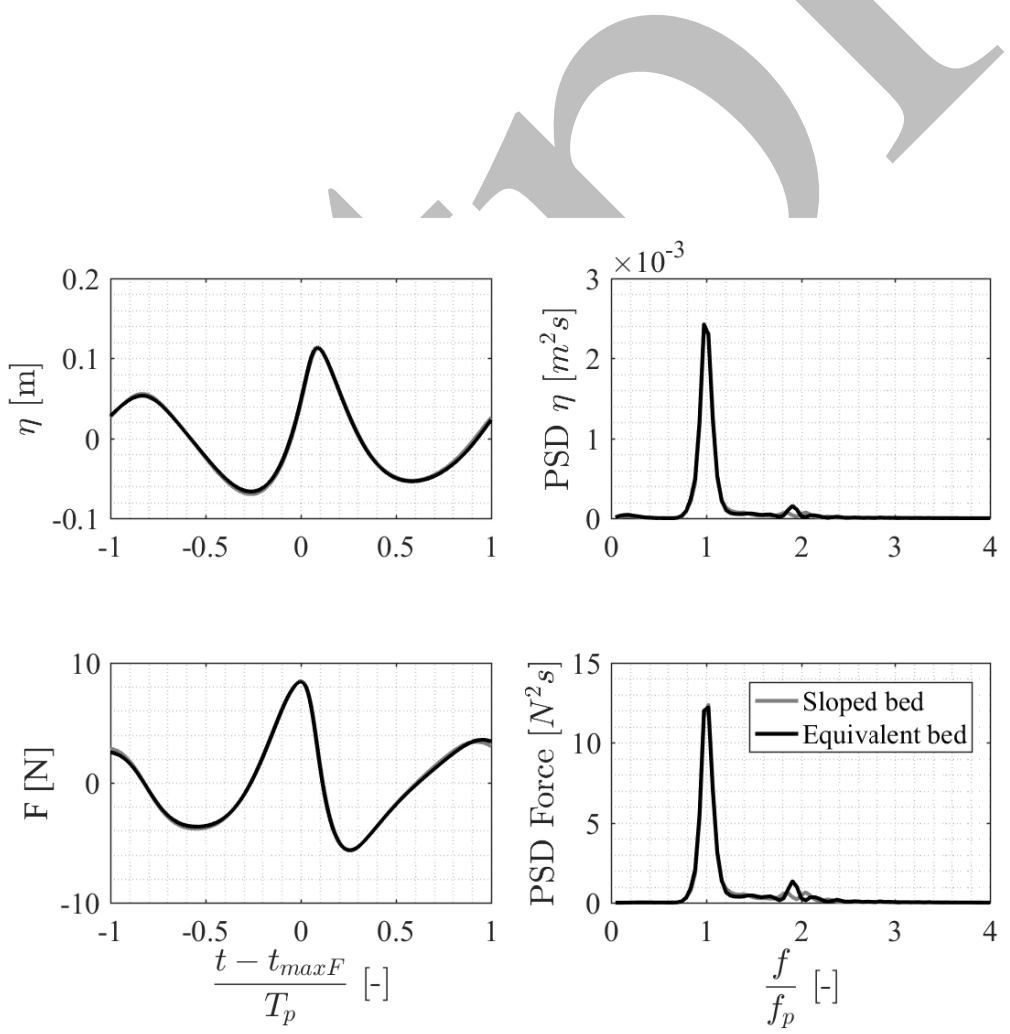

Figure A.32: Free surface elevation and inline force time series and power spectral density for numerical results of a case in sea state 4 with $F_{\text {target }}=1.2 \cdot \rho g h R^{2}$ with sloped bed and the equivalent flat bed. 

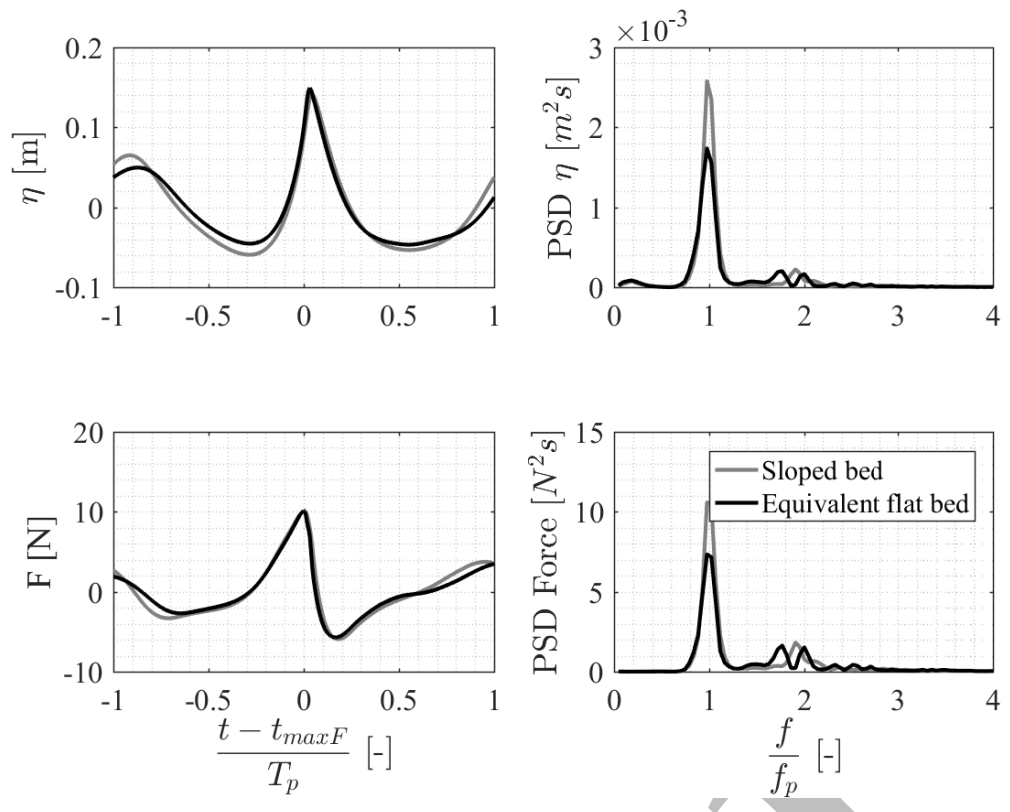

Figure A.33: Free surface elevation and inline force time series and power spectral density for numerical results of a case in sea state 6 with $F_{\text {target }}=1.7 \cdot \rho g h R^{2}$ with sloped bed and the equivalent flat bed.
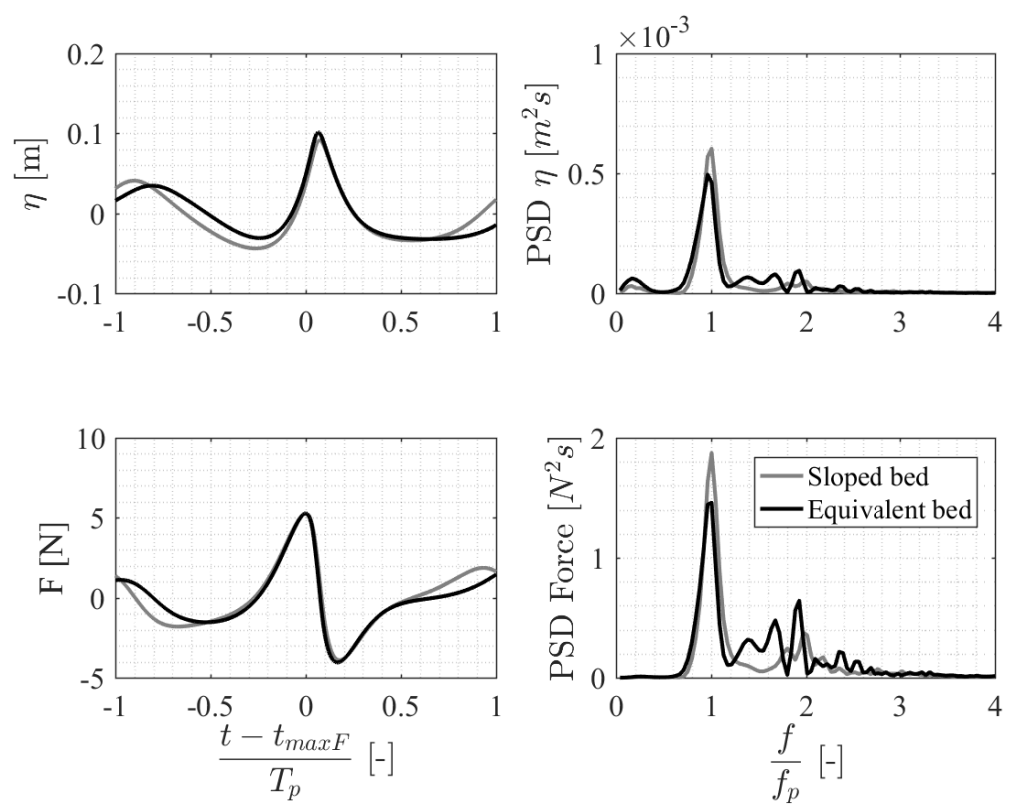

Figure A.34: Free surface elevation and inline force time series and power spectral density for numerical results of a case in sea state 7 with $F_{\text {target }}=1.5 \cdot \rho g h R^{2}$ with sloped bed and the equivalent flat bed. 

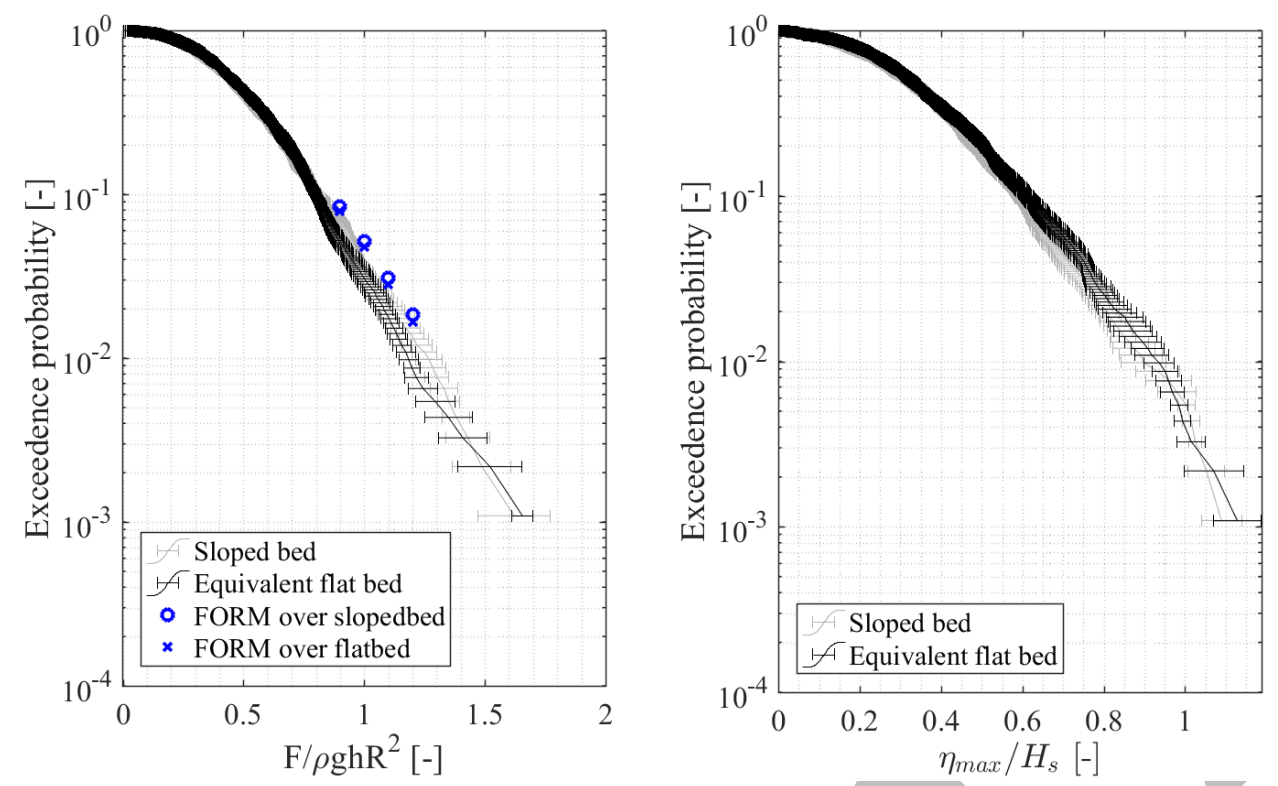

Figure A.35: Exceedance probabilities relative to the non-dimensional maximum inline force (left hand side plot) and maximum crest height (right hand side plot) for sea state 4. The results from FORM calculations are also included in the left hand side plot for sloped bed and equivalent flat sea bed.
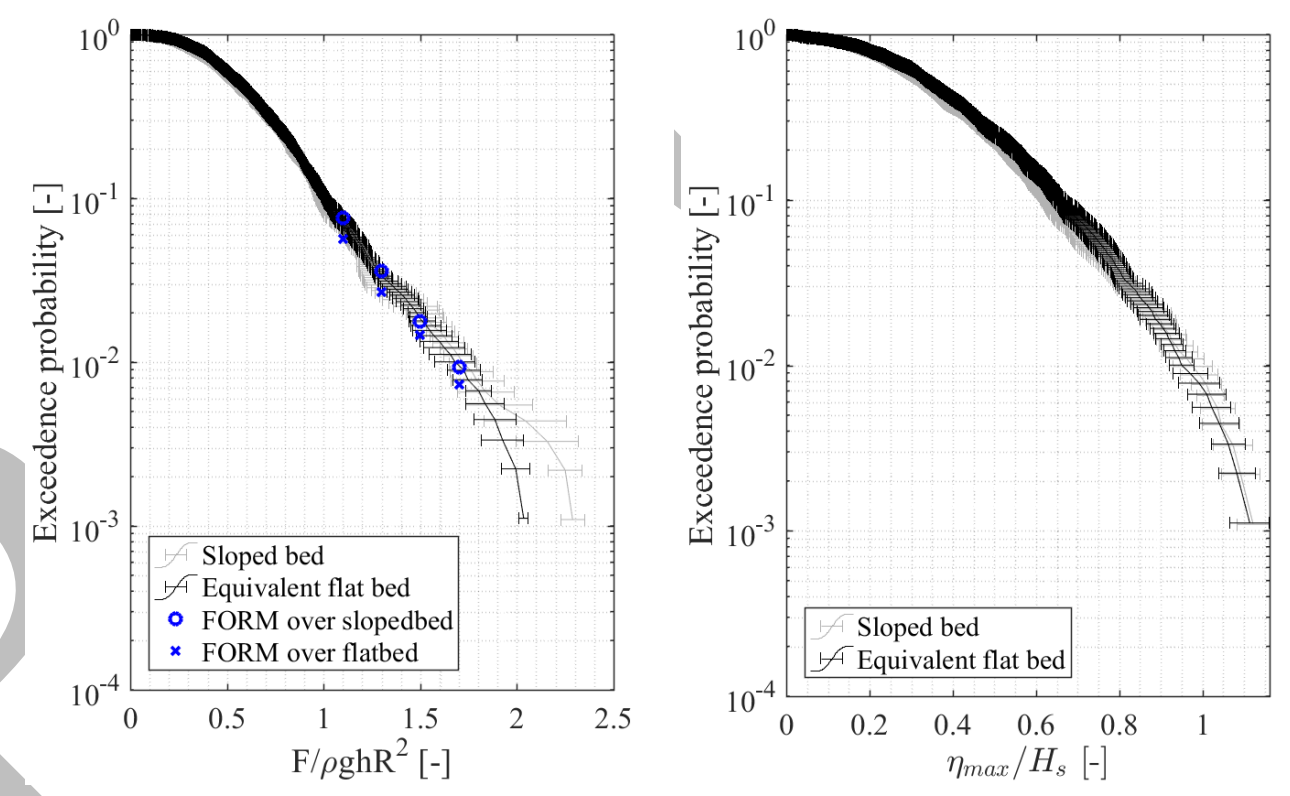

Figure A.36: Exceedance probabilities relative to the non-dimensional maximum inline force (left hand side plot) and maximum crest height (right hand side plot) for sea state 6 . The results from FORM calculations are also included in the left hand side plot for sloped bed and equivalent flat sea bed. 

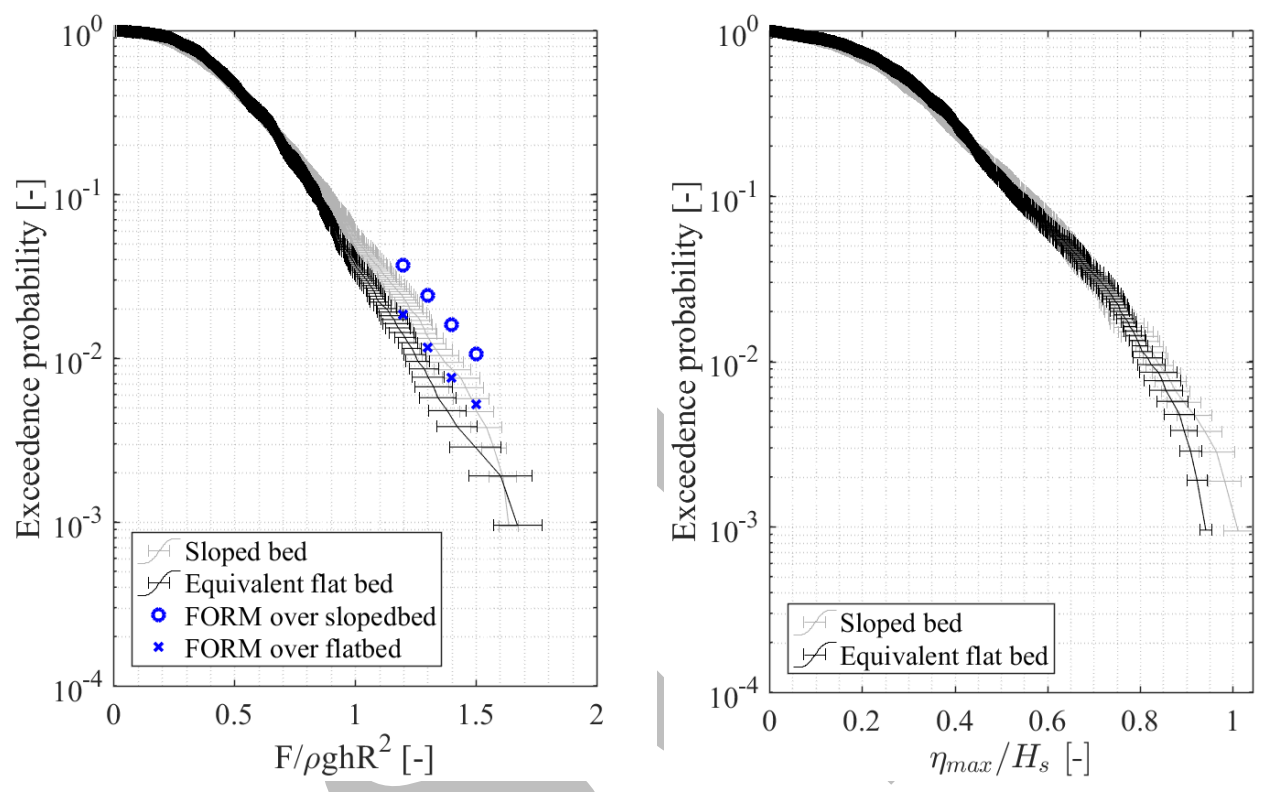

Figure A.37: Exceedance probabilities relative to the non-dimensional maximum inline force (left hand side plot) and maximum crest height (right hand side plot) for sea state 7. The results from FORM calculations are also included in the left hand side plot for sloped bed and equivalent flat sea bed.
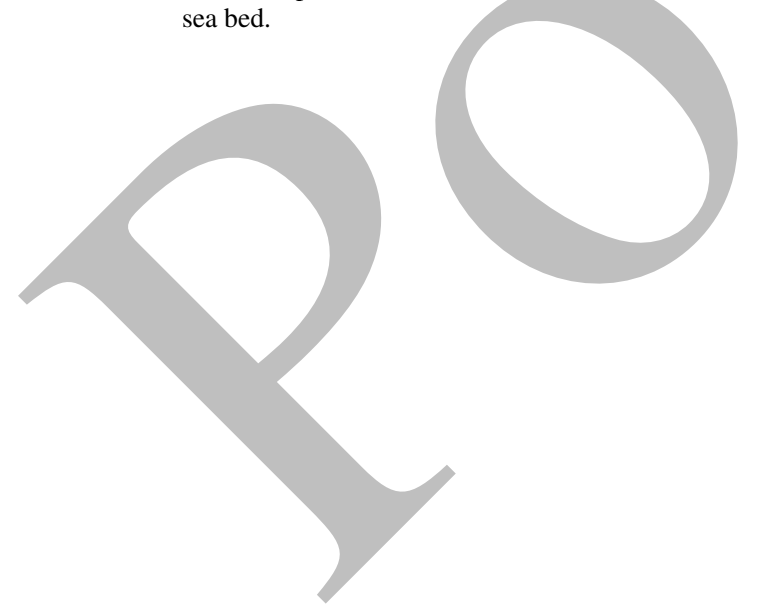